\title{
Ecologie des Leishmanioses dans le sud de la France
}

\author{
1. - Les Phlébotomes * \\ Echantillonnage - Ethologie
}

\author{
par J.-A. RIOUX, Y.-J. GOLVAN, H. CROSET, R. HOUIN, \\ B. JUMINER, O. BAIN et S. TOUR
}

\section{SOMMAIRE}

GÉNÉRALITÉs.

MÉTHOdES D'ÉCHANTILLONNAGE.

1. Capture manuelle diurne, dans les anfractuosités naturelles ou artificielles.

2. Capture manuelle nocturne à la lampe.

3. Capture manuelle sur appât humain.

4. Capture au piège-moustiquaire.

5. Capture au piège adhésif.

6. Capture au piège lumineux.

7. Capture au piège à $\mathrm{CO}_{2}$.

RÉGIONS PROSPECTÉES.

ETUde ANALYTIQUe.

1. Phlebotomus minutus Rondani.

2. Phlebotomus papatasi (Scopoli).

3. Phlebotomus mascittii Grassi.

4. Phlebotomus perniciosus Newstead.

5. Phlebotomus ariasi Tonnoir.

ETUde SYNTHÉtique - COROLlaires ÉPIDÉMIOLOGIQUes.

1. Arguments indirects.

2. Arguments directs.

* Collaboration technique: A. Brès, M. Maistre et A. Dumas. 
L'Ecologie, Science biologique, prend à l'heure actuelle une importance sans cesse croissante. Elle suppose en effet un mode de penser à la fois original et rigoureux qui conduit, dans l'étude des relations * organismes-milieux » à toujours plus de précision et d'objectivité. Elle est par excellence la « Science explicative ».

Ainsi, dans le cadre plus étroit des affections transmissibles, n'est-il pas étonnant de la voir placée à l'avant-garde des « méthodes d'approche ».

Renouvelant l'épidémiologie traditionnelle, elle permet en effet une interprétation beaucoup plus rigoureuse des « complexes pathogènes »: la maladie, conçue comme état d'équilibre entre germes, hôte et milieu, perd, ipso facto, son caractère artificiel pour s'intégrer dans les écosystèmes dont elle constitue habituellement un élément régulateur.

De fait, en matière de "cycle ", les études sont centrées actuellement, moins sur les agents causaux eux-mêmes que sur les facteurs extrinsèques qui conditionnent l'implantation, l'épanouissement et la permanence des zoonoses. Parmi celles-ci, les maladies transmissibles, c'est-à-dire à germes hétérogènes, occupent une place privilégiée. Qu'elles soient parasitaires, bactériennes ou virales, elles comportent en effet un certain nombre d'intermédiaires vivants (vertébrés réservoirs de virus, arthropodes vecteurs) ayant leur éthologie propre, tout en entretenant des rapports de coaptation suffisamment étroits pour permettre la « réussite» du germe pathogène.

Dès lors, correctement placée dans la biosphère, l'endémie y occupe une aire géographique parfaitement délimitée, et définie par un ensemble de facteurs bioclimatiques, écologiques et faunistiques, dont l'intégration conditionne ce qu'il est convenu d'appeler le « foyer naturel d'infection » (K. F. Meyer, 1941 ; U. M. Rall, 1958). Tel est le cas des arboviroses, des rickettsioses, des borellioses régionales, des pasteurelloses, des trypanosomoses et plus particulièrement des leishmanioses, objet du présent mémoire.

\section{$* *$}

C'est essentiellement dans cet esprit que nous présentons les premiers résultats de notre enquête sur les « vecteurs habituels » des leishmanioses, c'est-à-dire les Phlébotomes. Cette enquête, menée depuis 1961, en Languedoc-Roussillon, a porté sur les cinq espèces suivantes :

Phlebotomus minutus Rondani, 1943.

Phlebotomus papatasi (Scopoli, 1786).

Phlebotomus mascittii Grassi, 1908.

Phlebotomus perniciosus Newstead, 1911.

Phlebotomus ariasi Tonnoir, 1921.

\section{METHODES D'ECHANTILLONNAGE}

Avant d'aborder tout autre chapitre, il est nécessaire de décrire de manière aussi précise que possible les diverses méthodes de chasse employées au cours de notre 
enquête. L'interprétation des résultats et, plus encore, les hypothèses corrélatives, dépendent en effet autant des procédés que des lieux de captures.

Au cours des dernières années, nous avons mis au point et éprouvé plusieurs techniques d'échantillonnage destinées à dépister les biotopes, à apprécier les densités et à préciser les cycles saisonniers des Phlébotomes du «Midi » méditerranéen. Au total, six types de méthodes ont été utilisés, à savoir (tabl. I) :

- la capture manuelle diurne, dans les anfranctuosités naturelles ou artificielles;

- la capture manuelle nocturne, à la lampe ;

- la capture manuelle nocturne, sur appât humain ;

- la capture au piège-moustiquaire ;

- la capture au piège adhésif ;

- la capture au piège lumineux ;

- la capture au piège à $\mathrm{CO}_{2}$.

\begin{tabular}{|c|c|c|c|c|c|c|c|c|c|c|c|c|c|c|c|}
\hline \multirow{2}{*}{. } & & \multicolumn{5}{|c|}{ Phlebotomus ariosi } & \multicolumn{5}{|c|}{ Phlebotomus perniciosus } & \multicolumn{4}{|c|}{ Phlebotomus minutus } \\
\hline & & $\sigma$ & ㅇ & $\begin{array}{c}\text { \% des } \\
\text { Q } \\
\text { pour } \\
\text { I'espece }\end{array}$ & $\begin{array}{c}\% \text { des } \\
3 \\
\text { espeices }\end{array}$ & $\begin{array}{c}\text { ropport } \\
\text { oriosi } \\
\text { pernicio- } \\
\text { sus }\end{array}$ & $\sigma$ & ᄋุ & $\begin{array}{c}\% \text { des } \\
\text { Q } \\
\text { pour } \\
\text { respéce }\end{array}$ & $\begin{array}{c}\% \text { des } \\
3 \\
\text { especes }\end{array}$ & $\begin{array}{c}\text { ropport } \\
\text { pernicig } \\
\text { sus } \\
\text { oriosi }\end{array}$ & ó & Q & $\begin{array}{c}\% \text { des } \\
\text { Q } \\
\text { pour } \\
\text { respèce } \\
\end{array}$ & $\begin{array}{c}\% \text { des } \\
3 \\
\text { espéces }\end{array}$ \\
\hline \multirow{5}{*}{ 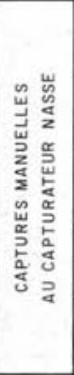 } & $\begin{array}{l}\text { APPAT } \\
\text { HUMAIN } \\
\text { (ext. noct.) }\end{array}$ & 0 & 471 & 100 & 99 & 99 & 0 & 4 & 100 & 1 & 1 & 0 & 0 & & $t$ \\
\hline & $\begin{array}{c}\text { APPAT } \\
\begin{array}{c}\text { ANIMAL (chiea) } \\
\text { (ext. noct.) }\end{array} \\
\end{array}$ & 3 & 126 & 98 & 100 & 100 & 0 & 0 & & 0 & 0 & 0 & 0 & & 0 \\
\hline & $\begin{array}{l}\text { HABITATIONS } \\
\text { (ext. noct.) }\end{array}$ & 356 & 2761 & 88 & 95 & 96 & 51 & 64 & 55 & 4 & 4 & 14 & 22 & 61 & 1 \\
\hline & $\begin{array}{c}\text { HABITATIONS } \\
\text { (int. noct el diurne) }\end{array}$ & 279 & 436 & 61 & 87 & 96 & 17 & 9 & 34 & 3 & 4 & 35 & 45 & 56 & 10 \\
\hline & $\begin{array}{l}\text { ROCHERS } \\
\text { MURS } \\
\text { (diurne) }\end{array}$ & 889 & 278 & 24 & 56 & 94 & 49 & 17 & 26 & 3 & 6 & 489 & 381 & 44 & 41 \\
\hline \multirow{2}{*}{ 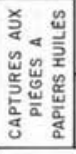 } & $\begin{array}{l}\text { PAPIERS } \\
\text { non.éclaites }\end{array}$ & 5666 & 455 & 7 & 28 & 97 & 205 & 12 & 6 & $t$ & 3 & 6046 & 9360 & 61 & 71 \\
\hline & $\begin{array}{l}\text { PAPIERS } \\
\text { ieloires }\end{array}$ & 238 & 321 & 57 & 79 & 99 & 2 & 1 & 33 & $t$ & $t$ & 18 & 121 & 87 & 20 \\
\hline \multicolumn{2}{|c|}{$\begin{array}{c}\text { CAPTURES OU PIEGE } \\
\text { ¿ } 22\end{array}$} & 3 & 25 & 89 & 97 & 100 & 0 & 0 & & 0 & 0 & 0 & 1 & 100 & 3 \\
\hline \multicolumn{2}{|c|}{$\begin{array}{c}\text { CAPTURES } \\
\text { Ou } \\
\text { "New Jersey mosq. trop" }\end{array}$} & 0 & 4 & 100 & 100 & 100 & 0 & 0 & . & 0 & 0 & 0 & 0 & : & 0 \\
\hline \multicolumn{2}{|r|}{ TOTAL } & 7434 & 4877 & 40 & 42 & 97 & 324 & 107 & 25 & 2 & 3 & 6602 & 9930 & 60 & 56 \\
\hline
\end{tabular}

Tableau I. - Tableau récapitulatif des captures réalisées dans le sud de la France de 1961

à 1966. Noter la nette prédominance de Phlebotomus ariasi par rapport à Phlebotomus perniciosus 
Parmi ces méthodes, certaines, de rendement faible ou nul, n'ont fait l'objet que de simples essais, tels le piège à anhydride carbonique ou le New Jersey mosquito trap. Les autres, d'efficacité suffisante, ont été retenues, mais utilisées dans des buts bien précis, soit d'échantillonnage quantitatif (piège adhésif), soit d'études éthologiques (piège lumineux, piège-moustiquaire) ou chorologiques (capture manuelle diurne).

\section{I. - Capture manuelle diurne, dans les anfractuosités naturelles ou artificielles}

Cette méthode, encore peu pratiquée en Europe, nous a donné d'excellents résultats. Certes, à l'opposé de la capture nocturne, elle ne permet pas toujours de récolter un grand nombre d'insectes par jour de piégeage et par personne (1), mais elle offre le grand avantage d'un échantillonnage plus précis, (gîtes de repos, gîtes de ponte), et plus éclectique (espèces non anthropophiles, grande proportion de mâles), ce en un temps relativement court et quelles que soient les conditions atmosphériques (vents, pluies, froid, etc...).

Cette méthode de chasse se pratique à l'aide d'un capturateur (2) de grande taille $(30 \mathrm{~cm})$ en verre épais ou en matière plastique, muni d'un tuyau souple d'aspiration (fig. 1).

Introduit à bout de bras dans la cavité (terriers, grottes, interstices de mur, barbacanes, etc...), le capturateur est poussé le plus loin possible, puis redressé de manière à explorer de proche en proche la partie haute du cul-de-sac, lieu de repos habituel des Phlébotomes. Ce faisant, une aspiration soutenue ne cesse de maintenir un violent appel

(1) Mentionnons cependant quelques captures massives comme dans l'observation suivante ( $n^{\circ}$ 253): «Le 26 juillet 1964 nous inventorions les gîtes ruraux de la région de Cessenon (Hérault) sur le versant Sud de la Montagne Noire orientale. A 12 h 30, nous explorons cinq barbacanes ménagées dans un mur de soutènement en bordure de la route D. 136, non loin du village de Lenthéric (alt. $160 \mathrm{~m}, \mathrm{t}^{\circ}$ ext. $29^{\circ} 5$, Hr. ext. $30 \%, \mathrm{t}^{\circ}$ des cavités $24^{\circ}$ ). En 15 minutes de piégeage (quatre personnes), nous capturons 103 Phlébotomes dont:

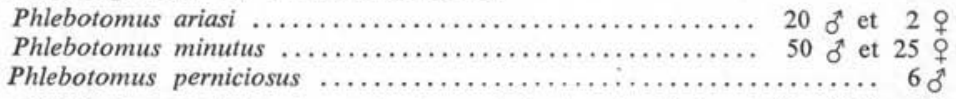

(2) En réalité, deux variantes de ces instruments ( $* \mathbf{A} »$ et « $\mathbf{B}$ ) ont été utilisées (fig 1):

$1^{\circ}$ Le modèle \& $\mathrm{A} »$, mis au point pour les cavités à orifice suffisamment large, est constitué par un simple cylindre de verre de $28 \mathrm{~cm}$ de longueur sur $4 \mathrm{~cm}$ de diamètre intérieur. Son extrémité apicale est terminée en forme de nasse dont louverture interne ne dépasse pas un centimètre de diamètre. Son extrémité proximale est obturée par un fort bouchon de caoutchouc sub-conique, lui-même traversé par un tube métallique de $1 \mathrm{~cm}$ de diamètre. Le segment apical de ce tube se prolonge à l'intérieur du cylindre de verre sur un à trois millimètres et se termine par une coiffe de grillage fin, ou, à défaut, de tissu à mailles serrées. A son extrémité libre s'adapte un tuyau de caoutchouc souple dont la longueur doit toujours rester supérieure à $90 \mathrm{~cm}$ (fig. $1 \mathrm{~A}$ ).

$2^{\circ}$ Le modèle \& $\mathrm{B} »$, utilisé dans les cas de fissures étroites (schistes) ou de cavités à petits orifices (terriers de reptiles) ne diffère du précédent que par son diamètre plus étroit $(2 \mathrm{~cm}) \mathrm{et} l a$ forme de son extrémité antérieure. Celle-ci est en effet munie d'un bouchon traversé par un tuyau de caoutchouc semi-rigide (longueur $10 \mathrm{~cm}$, diamètre $1 \mathrm{~cm}$ ) taillé en biseau à son extrémité (fig. $1 \mathrm{~B}$ ).

En pratique, nous utilisons surtout la variante « $\mathrm{A}$ » dont le rendement, dans la région méditerranéenne tout au moins, est nettement supérieur. 
d'air à l'orifice de la nasse. Le rendement de l'appareil dépend à la fois des mouvements communiqués à son extrémité et de la puissance du courant. Au surplus, un parfait synchronisme de ces deux temps est nécessaire, car l'Insecte, au repos dans une cavité

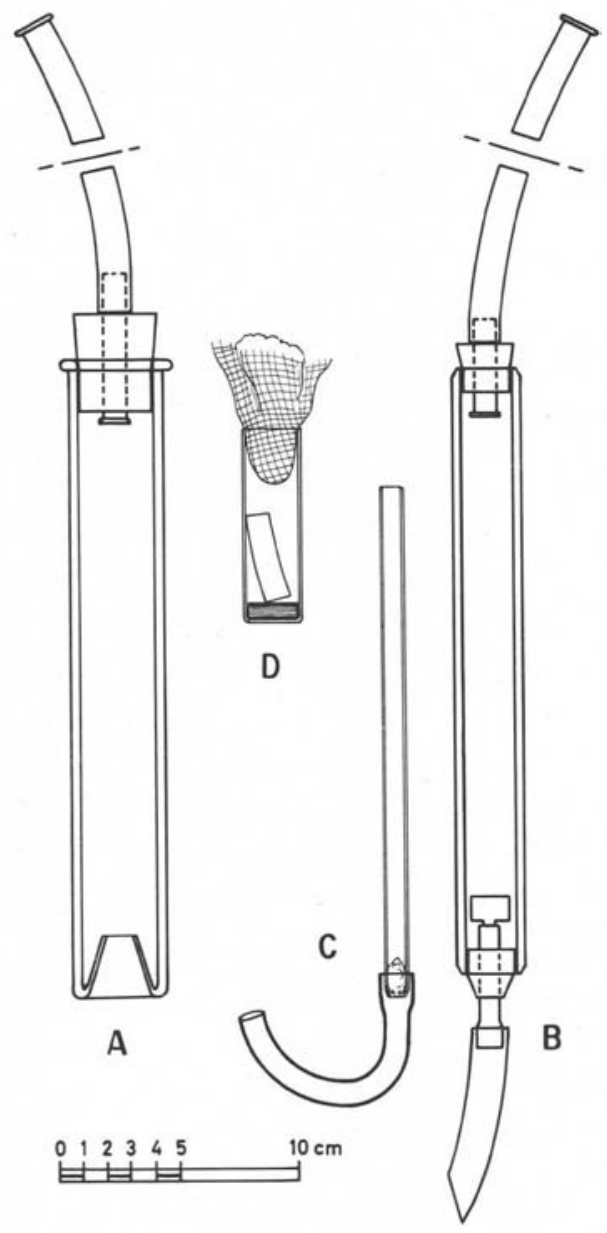

FIG. 1. - Matériel de capture. AB : deux modèles d'aspirateur-nasse. C: tube de prélèvement (pouvant pénétrer dans le capturateur A). D: tube de transport, de ponte et

obscure et humide, tend à rester plaqué contre les parois (temps de décrochage) et possède une agilité et une puissance de vol suffisante pour résister aux dépressions trop faibles (temps d'aspiration). 


\section{II. - Capture manuelle nocturne à la lampe}

Pour être efficace, cette chasse doit débuter dès le crépuscule et se poursuivre pendant les heures chaudes de la nuit, jusqu'aux environs de 24 heures.

Elle se pratique le plus souvent à l'extérieur, parfois à l'intérieur des habitations, à l'aide d'une lampe torche et d'un capturateur analogue au précédent, mais dont l'orifice interne de la nasse ne dépasse pas $0,5 \mathrm{~cm}$.

Il est indispensable, pour obtenir un rendement maximal, de se placer devant une paroi lisse et, si possible, de couleur claire (porte, fenêtre, mur crépi, falaise, etc...). Lorsque la chasse a commencé, il n'est pas recommandé de changer de poste car les Phlébotomes arrivent par « bouffées » et souvent d'une assez grande distance (3), attirés autant par la lumière que par le piégeur qui fonctionne alors comme appât. Habituellement l'insecte se pose en marge de la surface éclairée; il est alors rapidement coiffé du capturateur cependant qu'une brusque aspiration le fait passer dans la nasse aussitôt après l'envol.

Correctement conduite, cette méthode est d'un excellent rendement (4). Toutefois, malgré ces avantages, ce procédé d'échantillonnage demeure très sélectif (faible proportion de mâles, monotonie des captures) et toujours dépendant des circonstances atmosphériques. Comme toute chasse à « l'affût », il ne permet d'exploiter qu'un petit nombre de localités par jour (une seule en général).

\section{III. - Capture manuelle nocturne sur appât humain}

Nous utilisons pour cette chasse un capturateur-nasse légèrement plus court $(15 \mathrm{~cm})$. Afin d'éliminer les causes d'erreurs dues au phototactisme, nous opérons dans l'obscurité totale et, le plus souvent, dans nos sacs de bivouac. Seuls le visage et les mains sont alors offerts aux piqûres. Le Phlébotome signale sa présence soit par un vrombissement discret, mais caractéristique, soit par une sensation de cheminement cutanée. Toujours dans l'obscurité, le piégeur applique l'orifice du capturateur à l'endroit de la piqûre et par une brève aspiration fait pénétrer l'insecte dans le piège. Immédiatement après

(3) La portée de vol des Phlébotomes est classiquement considérée comme faible. Toutefois, parmi les espèces de la faune française, Phlebotomus ariasi paraît doué d'un pouvoir de dispersion non négligeable. Lors de nos captures nocturnes nous avons souvent observé des individus se déplaçant rapidement, en droite ligne, à plusieurs mètres au-dessus de nous.

(4) Un exemple du haut rendement de cette méthode nous est apporté par l'observation suivante $\left(n^{\circ}\right.$ 201). \& Le 12 août 1964, nous prospectons les abords immédiats de La Borie-Nouvelle (Hérault) petit village des avant-monts du Caroux (alt. $500 \mathrm{~m}$ ). A $21 \mathrm{~h}$, deux d'entre nous prennent place devant une maison dégagée et orientée plein Sud $\left(\mathrm{t}^{\circ} 17^{\circ} 5, \mathrm{Hr}\right.$. $55 \%$, vent nul). Après une heure de chasse il est décompté 446 Phlébotomes. Sur ce total, 160 q non identifiées sont inoculées extemporairement au Hamster par lots de 10. Le reliquat, transporté au laboratoire et déterminé, se compose de :

Phlebotomus ariasi

$26 \delta^{*}$ et 258 우

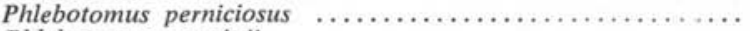

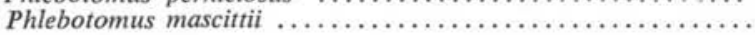

1 우 
chaque capture, il est recommandé d'obturer l'orifice de la nasse à l'aide d'un petit tampon de coton. Une certaine habitude (repérage, synchronisme des mouvements) est nécessaire pour obtenir un bon rendement.

Contrairement aux méthodes précédentes, la capture nocturne sur appât humain présente un intérêt épidémiologique tout particulier. Sa sélectivité permet en effet de ne capturer que les seules espèces piquant effectivement l'homme à l'exception d'espèces simplement attirées par la perspiration cutanée ou par le flux lumineux de la lampe de poche (cf. capture manuelle à la lampe).

\section{IV. - Capture au piège-moustiquaire}

Cette technique de piégeage utilise une ou plusieurs moustiquaires tendues à l'extérieur ou à l'intérieur pendant une série de nuitées. Après introduction de l'hôte-appât, un côté de la moustiquaire est relevé à l'aide d'un cadre de bois standard, de manière à ménager une ouverture de dimensions constantes. Suivant les cas, les Phlébotomes sont récupérés à intervalles réguliers ou en fin de nuit.

Cette méthode, mise au point au cours des années 1965-1966, a permis, outre les captures massives destinées à l'expérimentation, l'étude du trophotropisme des différentes espèces de Phlébotomes vis-à-vis des trois principaux hôtes naturellement infestés (Homme, Chien, Renard).

\section{V. - Capture au piège adhésif}

Ce mode de capture, basé sur les propriétés engluantes de l'huile de Ricin, est connu depuis les importants travaux des épidémiologistes russes Y. P. Vlasov (1931), W. et P. Pétrisheva (1935) au Turkestan (5).

Les pièges sont essentiellement constitués de feuilles de papier imbibées d'huile de Ricin et suspendues à un fil ou maintenues rigides par un support de bois (fig. 2). En pratique, malgré leur simplicité, la préparation et la pose de ces pièges demandent un certain nombre de précautions que nous résumerons brièvement :

Du papier, de teinte blanc mat, d'épaisseur faible, mais assez rigide (vélin glacé $72 \mathrm{~g}$ ) est découpé en feuillets carrés de $20 \mathrm{~cm}, 14 \mathrm{~cm}, 7 \mathrm{~cm}$ et $3 \mathrm{~cm}$ de côté. L'huile de Ricin, purifiée (huile officinale) de manière à réduire son pouvoir attractif sur les prédateurs, doit imprégner le papier en profondeur jusqu'à le rendre transparent. Pour ce faire il est nécessaire d'enduire successivement les deux faces. A cet usage, un rouleau de peintre peut être avantageusement utilisé. Les papiers, préalablement placés sur

(5) Ultérieurement, ce procédé a été utilisé avec succès par R. Kirk et D. J. Lewis (1940, 1946, 1951), puis par L. W. Quate (1964) en République du Soudan ; par N. L. Latishev et A. P. Kryukova (1941) en Russie; par L. Parrot et R. Durand-Delacre (1948), puis R. Durand-Delacre et Y. Menin (1953) en Algérie ; par J.A. Rioux et coll. (1965) en Tunisie; par G. Lupasco, P. Dancesco et N. Cheles (1965) en Roumanie. 
leur support (tige de roseau fendue, guirlande), sont stockés à plat. Une marque est inscrite sur l'une des faces de manière à orienter le piège dans les cavités (marque à l'extérieur).

Sur le terrain, les pièges sont introduits profondément dans les anfractuosités (grottes, fissures, barbacanes, etc...) et camouflés si nécessaire (bords de route, agglomérations), par des touffes d'herbes ou fragments de rochers. Ils peuvent être relevés dès le lendemain et jusqu'à un mois après la pose (6). Ils sont alors groupés, par station,

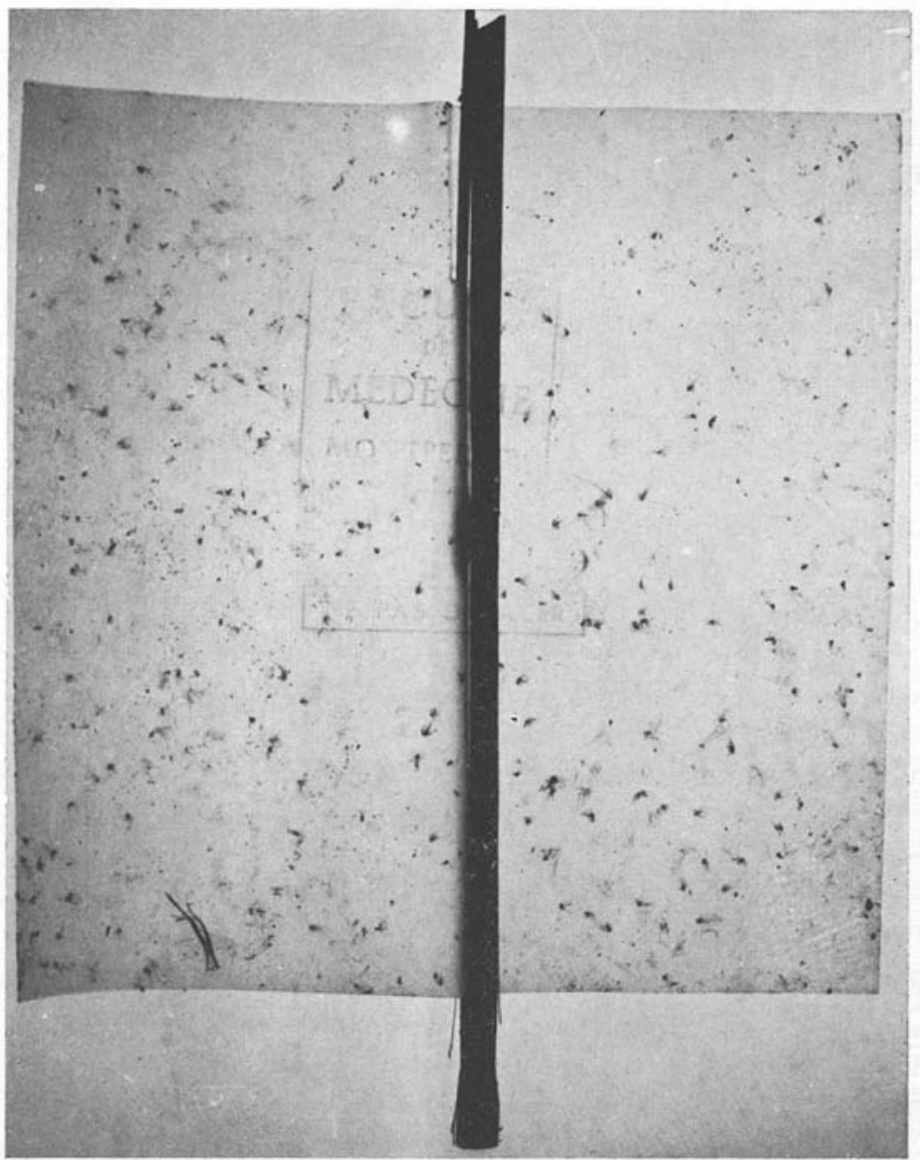

FIG. 2. - Un papier huilé $(20 \times 20 \mathrm{~cm})$ ayant capturé 163 Phlébotomes (Phlebotomus ariasi $3 q$ et 8 o , Phlebotomus minutus 72 et 80 ठ) en 15 jours de piégeage dans une barbacane (Caroux, Hérault, $230 \mathrm{~m}, \mathrm{n}^{\circ} 23^{\text {bis }}$ )

(6) Le « temps d'efficacité » de ce piège est variable suivant les régions, les saisons et les biotopes. Ainsi au Sahara tunisien, en juillet 1966, les papiers se desséchaient en 24 heures alors qu'en France, au mois d'octobre, l'huile persistait sans altération pendant les trois semaines de piégeage. Cette variabilité doit être prise en considération lors de la comparaison statistique des résultats. 
dans une chemise de papier sulfurisé ; leur conservation est ainsi assurée pour plusieurs jours. Toutefois, lors des longues prospections, les récoltes doivent être dépouillées et classées le soir même.

Repérés par transparence, sous une forte lumière, les Phlébotomes sont prélevés au pinceau imbibé d'alcool à $95^{\circ}$ et conservés en tube. Un séjour de 48 heures environ dans l'alcool fort est souvent nécessaire pour dissoudre totalement l'huile.

Au cours de notre enquête (7), nous avons utilisé les papiers huilés dans trois éventualités :

$1^{\circ}$ Durant les missions itinérantes, lorsque le parcours peut être effectué dans les deux sens. Les pièges sont placés à l'aller et relevés au retour.

$2^{\circ}$ A la halte du soir : la méthode des papiers huilés complète les autres méthodes de chasse. Elle apporte, en particulier, d'intéressantes indications mésologiques et éthologiques.

$3^{\circ}$ Dans les zones d'endémie leishmanienne: ici les pièges sont disposés suivant plusieurs «itinéraires-transects » établis en fonction de critères épidémiologiques et écologiques (8). Ainsi dans les départements de l'Hérault, du Gard et des Bouches-duRhône, trois transects (oriental, occidental et juxta-littoral) ont été mis au point puis exploités (1965 et 1966) :

Transect Est (fig. 3).

Ce transect, qui permet d'échantillonner les secteurs moyen et supérieur du bassin de l'Hérault, s'inscrit essentiellement dans le complexe forestier des Cévennes méridionales, c'est-à-dire la Chênaie d'Yeuse de plaine, la Chênaie d'Yeuse montagnarde, la chênaie caducifoliée et la Hêtraie. Partant de la côte $50 \mathrm{~m}$ à Montpellier, il atteint $1.230 \mathrm{~m}$ aux granites de l'Espérou après avoir traversé les « garrigues » crétacées, les basses montagnes jurassiques, le bassin sous-cébennique du Vigan (calcaires géorgiens et schistes ordoviciens) et les contreforts schisteux des Cévennes (Arcadien et Stéphanien). En pratique, ce transect comprend deux itinéraires, Sud et Nord, totalisant 64 stations pour $180 \mathrm{~km}$ de parcours.

(7) En 1965 il a été posé 7.813 pièges correspondant à $235 \mathrm{~m}^{2}$ de papier et 65 litres d'huile de ricin $\left(138 \mathrm{ml} / \mathrm{m}^{2}\right)$. Pour chaque espèce, le rendement moyen s'est établi comme suit :
Phlebotomus
Phlebotomus

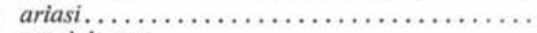
32 individus $/ \mathrm{m}^{2}$
2 individus $/ \mathrm{m}^{2}$

Sur les $235 \mathrm{~m}^{2}$ de pièges mis en place dans les gîtes, $159 \mathrm{~m}^{2}$ seulement ont été relevés et dépouillés (pertes : $32 \%$ ).

(8) En moyenne, durant la période d'activité des Phlébotomes, chaque transect est échantillonné toutes les trois semaines.

Mise en application en 1965, de juin à novembre, cette méthode nous a permis de récolter 20.234 Phlébotomes dont :
Phlebotomus ariasi

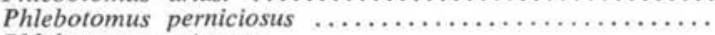
4.714
$173 \sigma^{\circ}$
Phlebotomus minutus
5.913
238 웅
5 웅
9.191 우 


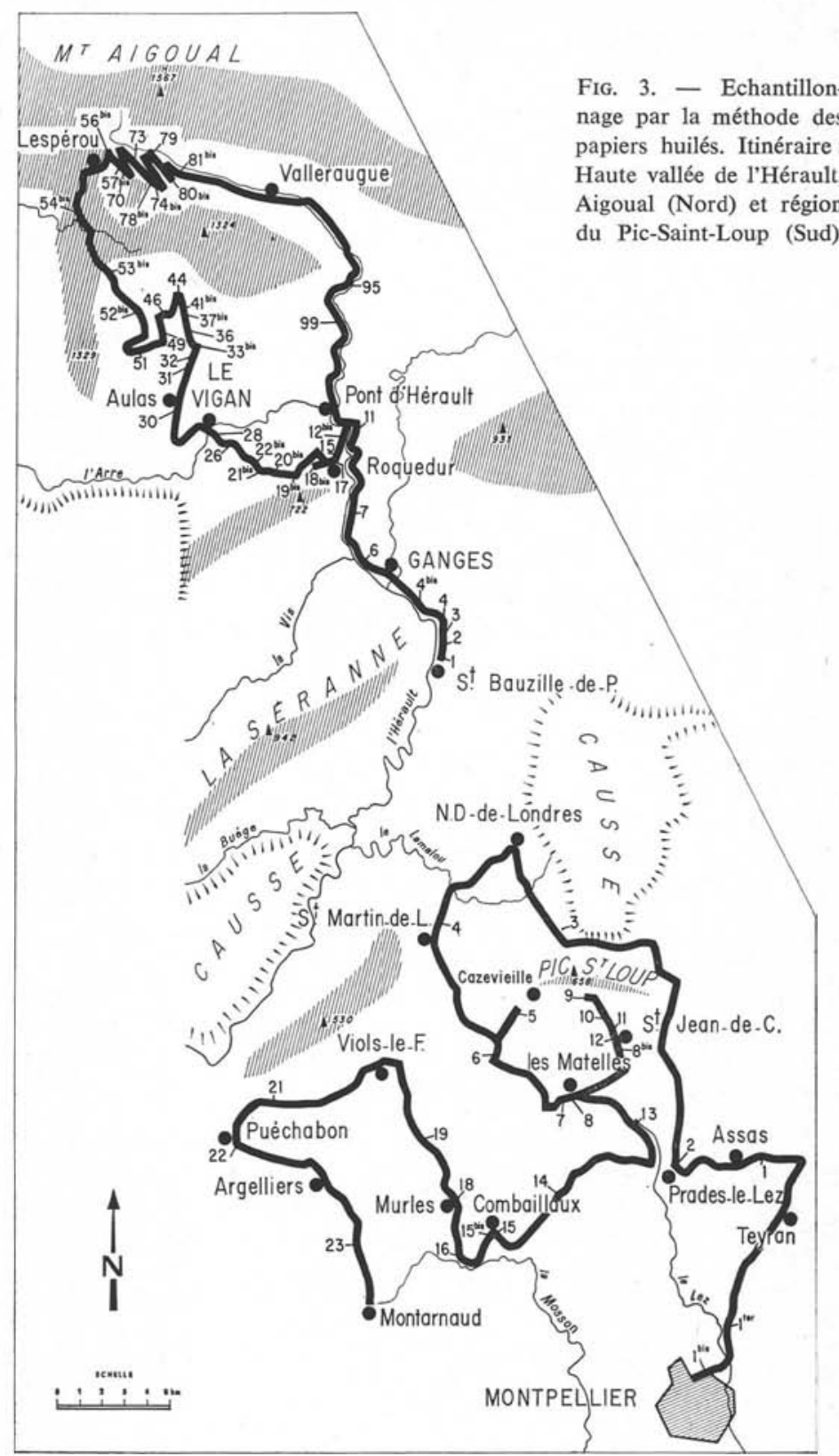




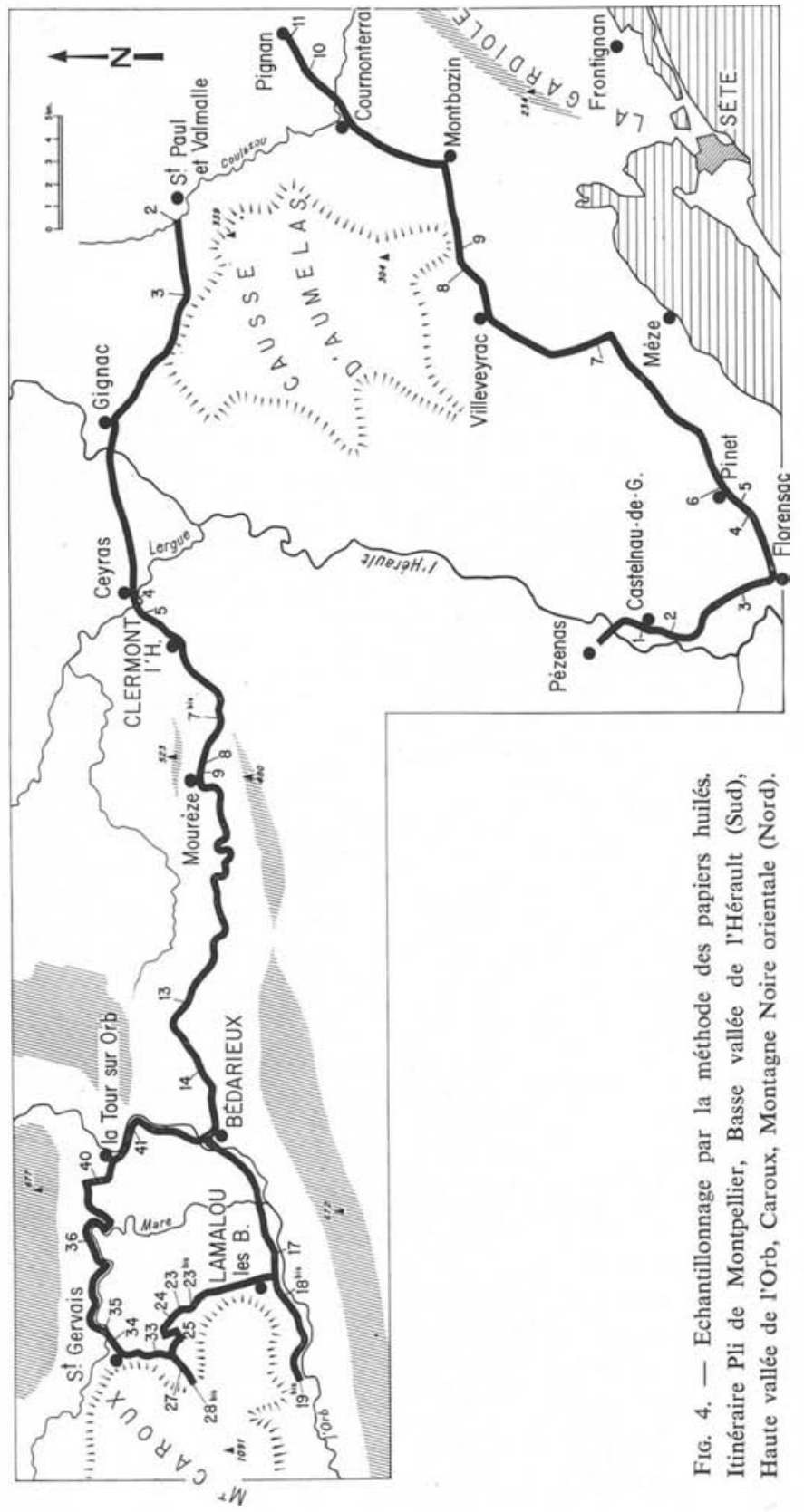




\section{Transect Ouest (fig. 4).}

Mieux que le précédent, le transect permet l'échantillonnage des garrigues littorales. Il intéresse l'important foyer leishmanien de la moyenne vallée de l'Orb et atteint $800 \mathrm{~m}$ dans le massif du Caroux. Partant de la région volcanique d'Agde, il traverse les alluvions récentes de la plaine biterroise, se poursuit dans le primaire sédimentaire de la Montagne Noire orientale (Cambrien) pour se terminer dans le métamorphique pré-cambrien de l'Espinouse. Il comprend également deux itinéraires, Sud et Nord, et totalise 37 stations pour $220 \mathrm{~km}$ de trajet.

\section{Transect juxta-littoral (fig. 5).}

Ce transect permet l'échantillonnage des basses plaines alluvionnaires et des marais littoraux. Il comprend, pour un trajet de $49 \mathrm{~km}$, cinq stations échelonnées depuis Sylvéréal en Petite Camargue jusqu'aux Salins-de-Badon, en Camargue orientale.

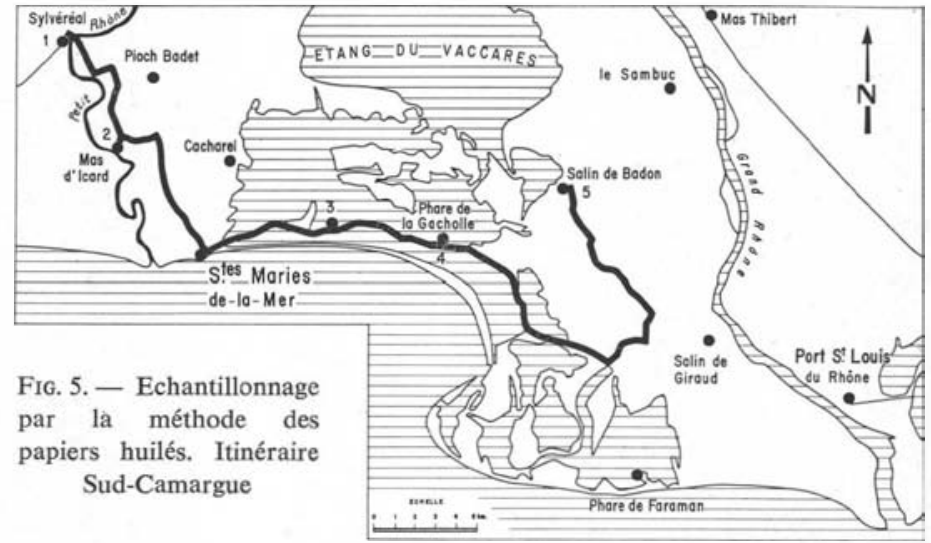

A l'instar de la chasse diurne au capturateur, la technique des papiers huilés permet d'inventorier les lieux de repos, voire les gîtes de pontes. Au surplus, elle se révèle infiniment plus intéressante dans l'échantillonnage quantitatif d'une région car elle réduit considérablement le coefficient personnel inhérent aux méthodes manuelles. Elle entraîne toutefois un grand nombre de manipulations et nécessite, dans le cas d'enquêtes importantes, la mise en place de deux équipes fonctionnant simultanément, l'une pour la capture, et le dépouillement, l'autre pour identification. A son passif notons également une certaine sélectivité portant, en particulier, sur les sexes. Il est ainsi curieux de constater que Phlebotomus ariasi et Phlebotomus perniciosus sont surtout représentés par les mâles (respectivement $95 \%$ ठ et $97 \% \delta^{*}$ ) alors que pour Phlebotomus minutus les femelles dominent sensiblement $\left(\begin{array}{lll}37 & \% & \delta^{*}\end{array}\right)$. Ces différences trouvent peut-être leur explication dans l'abondance des reptiles et la rareté des mammifères dans les biotopes inventoriés par cette méthode. 


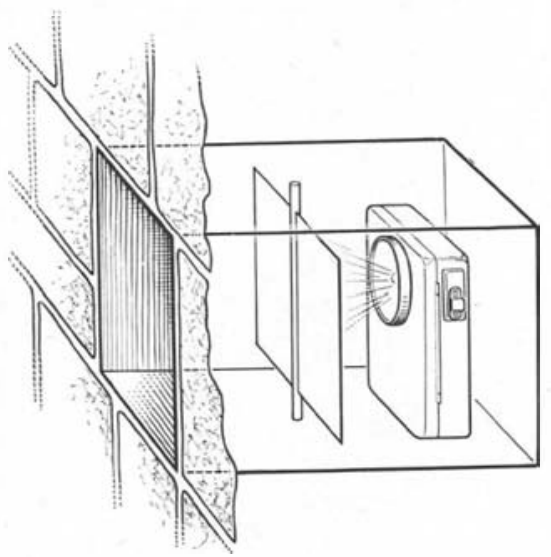

Fig. 6. - Papier huilé de $20 \times 20$ mis en place dans une barbacane et éclairé par une lampe de poche

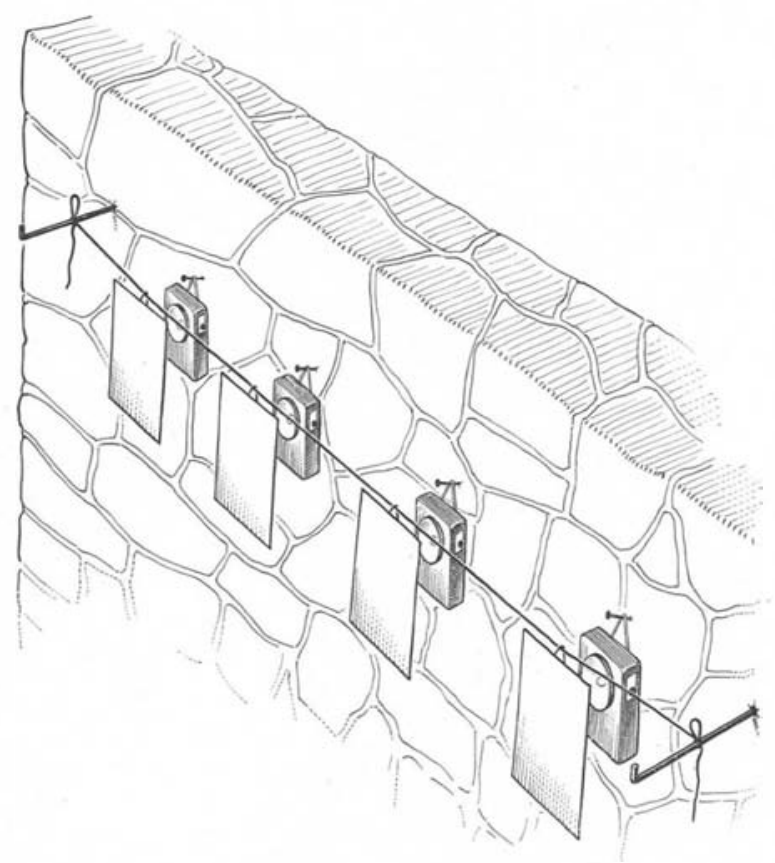

Fig. 7. - Guirlande éclairée, tendue contre un mur 


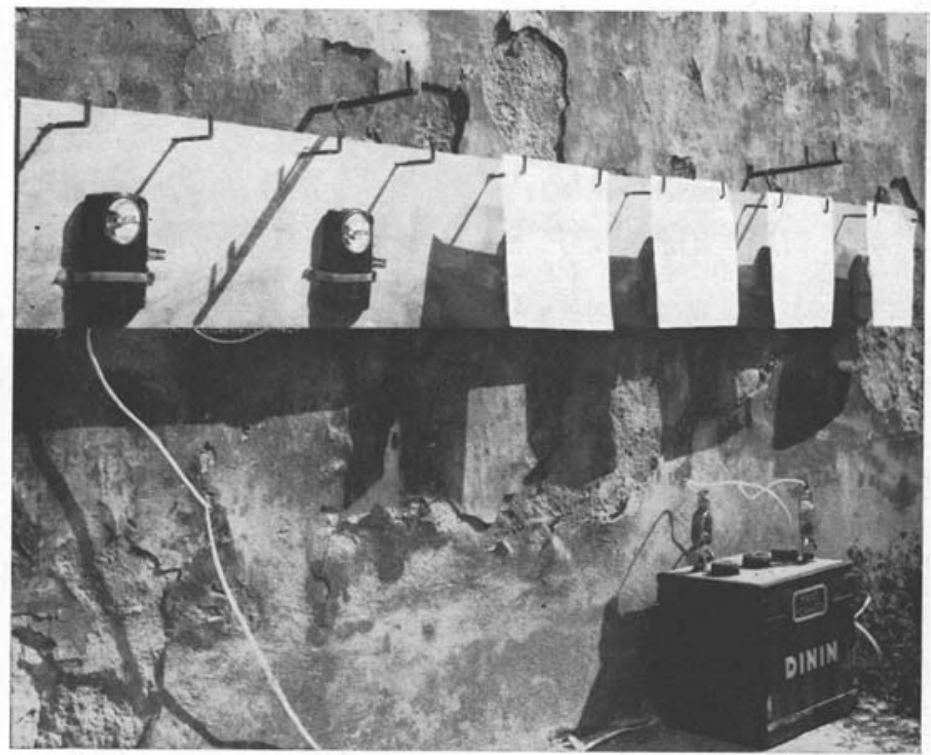

FIG. 8. - Montage pour l'étude du phototactisme des Phlébotomes. Les deux premiers papiers huilés n'ont pas été placés, de manière à mettre en évidence les lampes
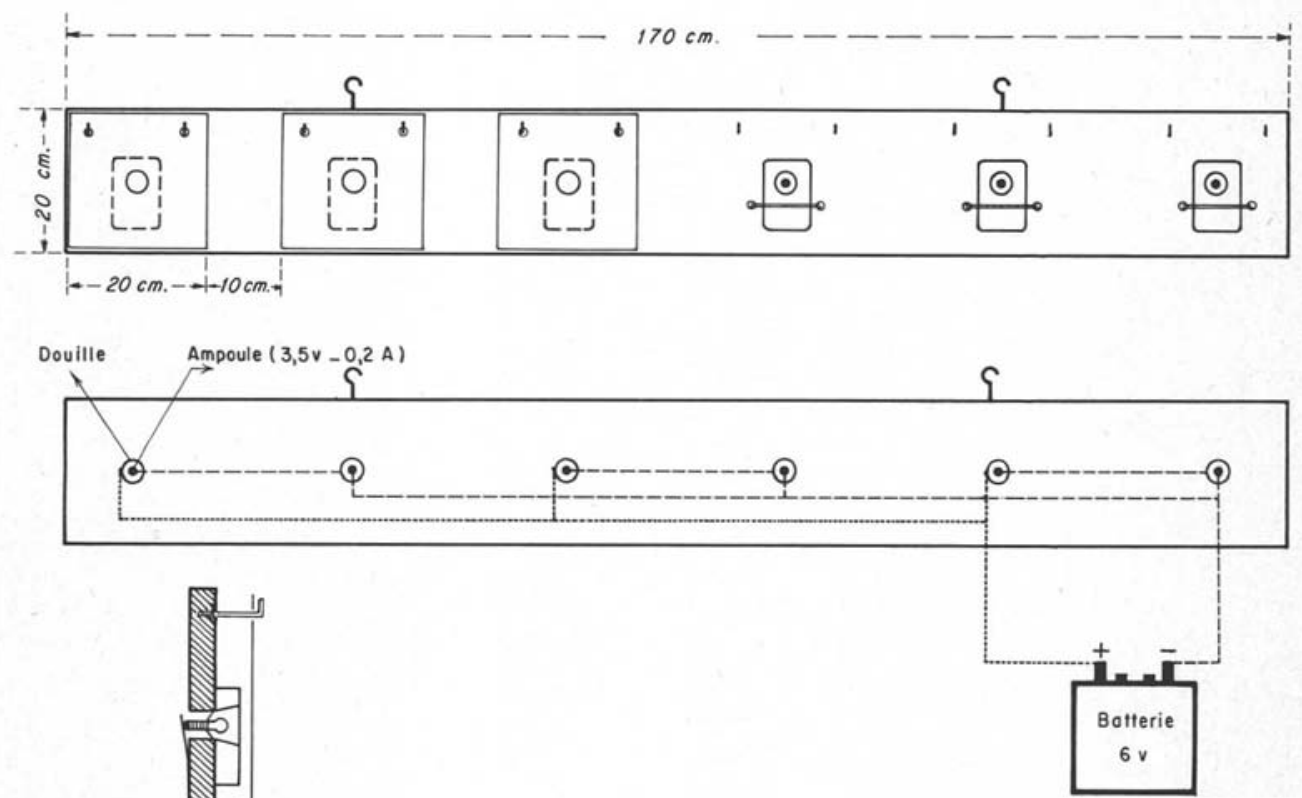

FIG. 9. - Schéma de montage du piège précédent 


\section{VI. - Capture au piège lumineux}

Dans le chapitre consacré au phototactisme de Phlebotomus ariasi nous rappellerons les difficultés inhérentes à ce mode de chasse. Insistons ici sur le fait qu'à elle seule, la source lumineuse ne saurait constituer un véritable piège. En effet, à l'inverse de beaucoup d'autres insectes (Lépidoptères, Diptères Cératopogonides et Chironomidés), les Phlébotomes demeurent rarement prisonniers du flux lumineux et semblent même repoussés à partir d'une certaine intensité. En bref, pour être efficace, un tel type de piège doit être non seulement attractif, c'est-à-dire doté d'une source de faible puissance, mais aussi contentif de manière à immobiliser définitivement les Phlébotomes après leur pénétration dans la zone éclairée.

Déjà la chasse nocturne contre les parois claires, constitue une illustration de cette méthode (attraction par la lumière réfléchie de la lampe de poche, contention par le capturateur-nasse). Plus intéressant est l'emploi du papier huilé comme système de contention (R. Kirk et D. J. Lewis, Soudan 1947).

En pratique, nous utilisons trois variantes de cette technique:

Variante A. - Une feuille de papier huilé $(20 \times 20 \mathrm{~cm})$ montée sur un support de bois, est introduite dans une anfractuosité (barbacane, creux de rocher) et éclairée, par derrière, à l'aide d'une lampe de poche (fig. 6). Dans le cas de prospection itinérante ce procédé permet d'augmenter le rendement du simple papier huilé mis en place le soir et retiré le lendemain.

Variante B. - Une guirlande, comportant quatre à sept feuilles de papier $(20 \times 20 \mathrm{~cm})$ imbibées d'huile de Ricin, est tendue horizontalement entre deux supports à $10 \mathrm{~cm}$ d'un mur. En arrière de chaque papier, si possible, est suspendue une lampe de poche qui projette la totalité de son flux lumineux sur la face postérieure de la feuille (fig. 7). Lors des périodes de pleine activité, ces dispositifs donnent d'excellents résultats (9).

Dans les deux cas, la méthode a l'avantage d'être simple et peu onéreuse. Elle ne nécessite aucun appareillage spécialisé et son rendement reste excellent.

Variante C. - L'intérêt épidémiologique des données recueillies par la variante B nous a amenés à perfectionner ce type de piège, de manière à standardiser autant que possible les résultats tout en diminuant son prix de revient.

(9) Tel est le cas de l'observation suivante $\left(n^{\circ} 407\right)$ : «Le 29 juillet 1965, à La Borie-Nouvelle (Hérault, alt. $500 \mathrm{~m}$ ), nous plaçons deux guirlandes de 5 feuilles $(20 \times 20 \mathrm{~cm})$ devant un mur de pierres sèches, en contrebas d'une vigne. La guirlande éclairée comporte quatre lampes de poche uniformément réparties. La guirlande témoin est placée en pleine obscurité à deux mètres de la précédente.

En une nuit de piégeage, nous capturons 263 Phlébotomes dont :

a) Guirlande éclairée :

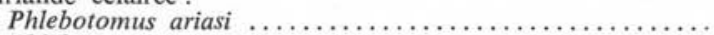

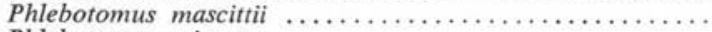

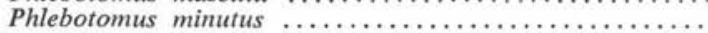

b) Guirlande témoin :

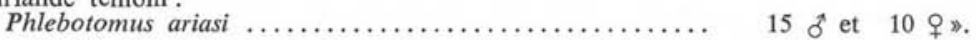

$$
\begin{aligned}
& 39 \text { o } 0 \text { et } 189 \text { 우 } \\
& 1 \text { ot } 10 \text { o } \\
& 15 \text { o } 10 \text { et } 10 \text { " }
\end{aligned}
$$


Le système que nous utilisons actuellement se compose d'un rectangle de contreplaqué de $170 \mathrm{~cm}$ de long sur $20 \mathrm{~cm}$ de large. Tous les $30 \mathrm{~cm}$ un réflecteur de lampe de poche (ampoule 3,5 volts) est fixé devant un orifice de $2 \mathrm{~cm}$ de diamètre permettant sa connection avec le circuit électrique monté au dos. Devant chaque lampe est suspendue une feuille de papier huilé $(20 \times 20 \mathrm{~cm})$. Un accumulateur de 6 volts, rechargé régulièrement par un groupe électrogène, alimente sans difficulté les pièges durant les six heures d'utilisation (fig. 8 et 9). Un coupe-circuit automatique permet d'éteindre le piège à l'heure désirée. Un piège identique mais dépourvu de montage électrique, sert de témoin.

Cette méthode de piégeage permet:

a) De juger du phototactisme d'une espèce, et par-là même du rôle éventuel joué par la lumière dans la pénétration des Phlébotomes à l'intérieur des habitations.

b) D'échantillonner rationnellement une localité grâce aux possibilités d'expression quantitative fournie par «l'unité piège » et la «nuit-piège » (10).

c) D'étudier les fluctuations journalières des populations de Phlébotomes par rapport aux variations des divers facteurs climatologiques. Cette dernière application est illustrée par les résultats de douze nuits de piégeages réalisées au cours de l'été 1966 :

\&Du 8 au 20 juillet 1966, nous prospectons le village de La Borie-Nouvelle (Hérault) situé à $500 \mathrm{~m}$ d'altitude, sur le versant méridional de la Montagne Noire orientale (Monts de Faugère). Ce site, construit au centre d'un hémicycle orienté «plein midi », ne comporte qu'une dizaine de maisons dont seule l'une d'entre elles est régulièrement habitée. Directement soumise aux influences marines (littoral à $36 \mathrm{~km}$ ), fortement insolée en raison de l'orientation et de la pente, efficacement protégée des vents du nord, violents et drastiques, La Borie-Nouvelle réalise les conditions optimales pour l'étude des variations journalières des populations de Phlébotomes.

A partir du 8 juillet, un piège lumineux «standard » est placé contre un mur de pierres sèches et mis en fonctionnement toutes les nuits, depuis le coucher de soleil jusqu'à 3 heures du matin. Un équipement météorologique sommaire (thermo-hygrographe) est installé à proximité du piège. En douze jours, 124 Phlébotomes sont capturés. L'étude de la variation journalière des captures montre une très étroite corrélation entre la densité des Phlébotomes d'une part, les températures moyennes, les orages et les caractéristiques des vents dominants d'autre part (fig. 10 et 15 ).

\section{VII. - Capture au piège à $\mathrm{CO}_{2}$}

Le pouvoir attractif du $\mathrm{CO}_{2}$ vis-à-vis des arthropodes hématophages est connu depuis longtemps. Utilisant cette propriété, de nombreux auteurs ont construit des pièges à haut rendement. Citons, en particulier, W. C. Reeves (U.S.A., 1961), pour les

(10) «L'unité-piège » correspond à la feuille de papier $\mathrm{n}^{\circ} 4(20 \times 20 \mathrm{~cm})$, offrant $800 \mathrm{~cm}^{2}$ de surface efficace. La «nuit-piège» correspond aux captures réalisées par l'unité-piège pendant une nuit. 
Culicidae et R. L. Nelson, puis R. H. Withsel et R. F. Schoeppner (U.S.A., 1964) pour les Ceratopogonidae.

FLUCTUATIONS JOURNALIERES DES POPULATIONS DE PHLEBOTOMES

EN FONCTION DES TEMPERATURES MOYENNES

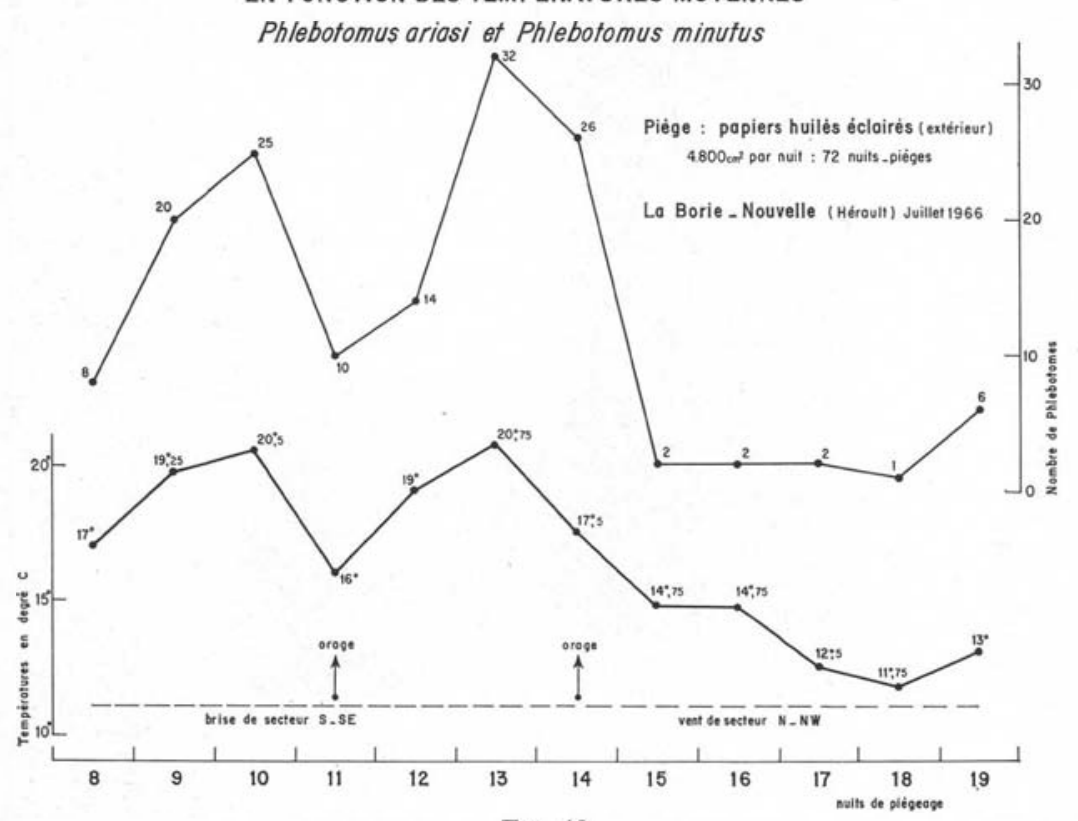

Fig. 10

A notre tour, nous avons tenté l'essai des pièges à $\mathrm{CO}_{2}$ pour la capture et l'échantillonnage des Phlébotomes. A cet effet, nous avons utilisé les deux dispositifs suivants:

\section{$1^{\text {er }}$ dispositif :}

Un seau cylindrique en matière plastique de $25 \mathrm{~cm}$ de diamètre est obturé par un disque de papier perforé sur toute sa surface par des trous de $3 \mathrm{~cm}$ de diamètre. Le disque et la face extérieure du cylindre sont badigeonnés d'huile de Ricin. Dans le piège est placé un pain de $2 \mathrm{~kg}$ de $\mathrm{CO}_{2}$ solide. Un piège identique mais dépourvu de $\mathrm{CO}_{2}$ sert de témoin.

\section{2 dispositif :}

A l'intérieur d'un cylindre métallique de $18 \mathrm{~cm}$ de diamètre et de $30 \mathrm{~cm}$ d'arête ouvert aux deux extrémités est glissé un cylindre de $12 \mathrm{~cm}$ de diamètre sur $15 \mathrm{~cm}$ d'arête obturé à la partie inférieure par un fond percé d'orifices de $1 \mathrm{~cm}$ de diamètre et muni 
à sa partie supérieure de trois pattes horizontales qui prennent appui sur le bord supérieur du cylindre extérieur. Le cylindre intérieur reçoit $2 \mathrm{~kg}$ de $\mathrm{CO}_{2}$ solide. Sur le cylindre extérieur est enroulée une feuille de papier qui le recouvre entièrement (fig. 11).

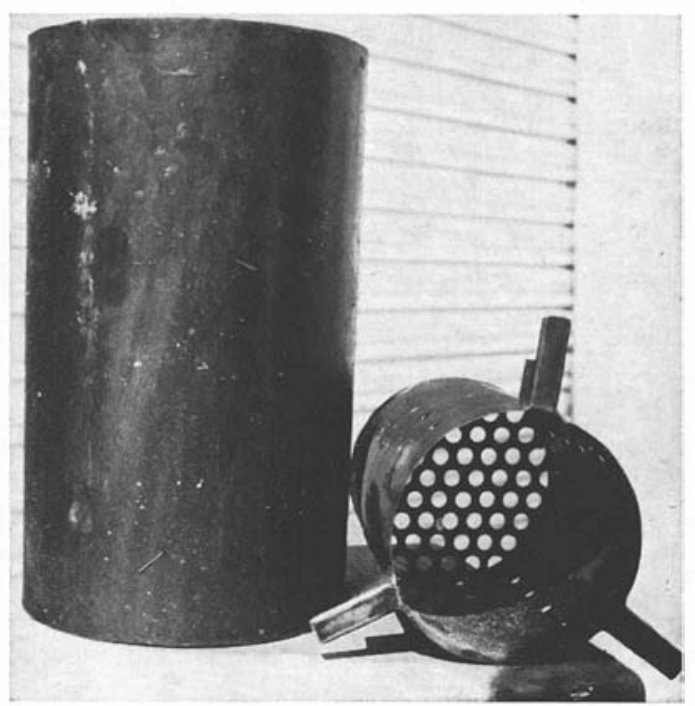

Fig. 11. - Les deux éléments du piège à anhydride carbonique. A droite, vue axiale du récipient à $\mathrm{CO}_{2}$ solide

\section{REGIONS PROSPECTEES}

Amorcée en 1961, poursuivie de 1962 à 1964, intensifiée en 1965 et 1966, notre enquête sur les vecteurs des Leishmanioses a couvert essentiellement la partie occidentale du foyer méditerranéen français, c'est-à-dire le secteur situé à l'ouest du Rhône. Ce faisant, nous avons prospecté les Basses-Cévennes, la Montagne Noire orientale, le versant méridional du massif Caroux-Espinouse, l'étage collinéen du Roussillon et des Albères. Toutefois la nécessité d'un inventaire comparatif nous a amenés à déborder largement sur les régions limitrophes, soit de type climatique méditerranéen (Corbières, Camargue, Bas-Languedoc, Haute et Basse-Provence) soit de types climatiques atlantique ou médio-européen (Mont-Lozère, Aigoual, Grands-Causses, Montagne Noire occidentale, Haut-Conflent, Bassin sous-pyrénéen). Nous avons ainsi échantillonné les départements suivants : 
Basses-Alpes

A!pes-Maritimes

Ardèche

Ariège

Aude

Aveyron

Bouches-du-Rhône

Drôme

Gard

Hautes-Alpes
Haute-Garonne

Hérault

Lot-et-Garonne

Lozère

Pyrénées-Orientales

Sarthe

Tarn

Var

Vaucluse

$\mathrm{Au}$ surplus, à titre comparatif, nous avons étendu quelque peu notre champ de prospection au-delà des frontières, en Italie (1964), en Espagne (1965) et en Tunisie (1966).

Au cours de cette enquête nous avons identifié 29.304 Phlébotomes se répartissant comme suit (tabl. II) :

TABLEAU II

Phlebotomus ariasi

Phlebotomus perniciosus ...................

Phlebotomus mascittii . . ...................

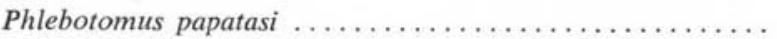

Phlebotomus minutus
$12.312(42,015 \%)$

$432(1,474 \%)$

$27(0,092 \%)$

$1(0,003 \%)$

$16.532(56,416 \%)$

\section{ETUDE ANALYTIQUE}

PHLEBOTOMUS (PROPHLEBOTOMUS) MINUTUS RONDANI, 1843

\section{Répartition géographique.}

L'aire géographique de Phlebotomus minutus minutus intéresse uniquement la bordure Nord du Bassin méditerranéen, du Portugal à la Grèce. En Afrique du Nord, il est remplacé par la sous-espèce parroti (Adler et Theodor, 1927) caractérisée par un nombre de dents cibariales plus élevé (70 à 85 contre 40 à 60). En Languedoc méditerranéen son maximum de densité s'observe entre 100 et 300 mètres d'altitude (fig. 12).

\section{Ethologie.}

\section{$1^{\circ}$ Comportement trophique.}

Les nombreuses observations, relatives au comportement trophique de Phlebotomus minutus, confirment sans ambiguïté sa tendance herpétophile. De fait, au cours de 
notre enquête et singulièrement à l'occasion de chasses nocturnes, nous n'avons jamais été attaqués par cette espèce, alors qu'à plusieurs reprises nous l'avons observée se gorgeant sur le Lézard des murailles.

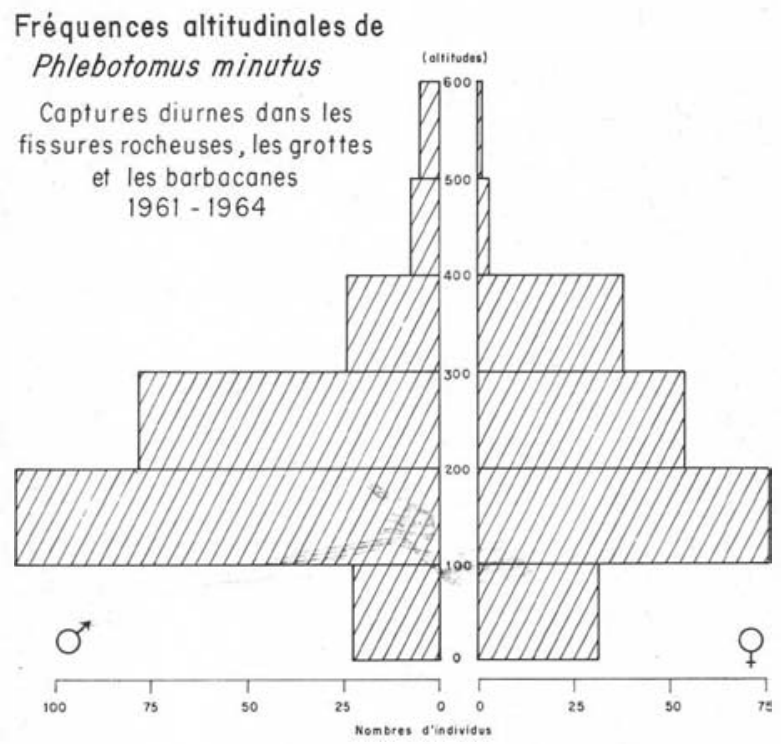

FIG. 12

Un tel comportement explique d'ailleurs le rôle joué par les représentants du groupe minutus dans la transmission de certains parasites sanguicoles aux Lacertidés. Ainsi, en Afrique du Nord, la sous-espèce parroti inocule au Gecko (Tarentola mauritanica) un Trypanosomatidé, Leishmania tarentolae Wenyon, 1921, primitivement confondu avec l'agent du Bouton d'Orient. L'infestation, étudiée expérimentalement par L. Parrot en 1935, procède non pas de la piqûre de l'insecte mais de son ingestion par le Reptile. En France, nous avons retrouvé le parasite dans le sang d'un Gecko provenant des environs de Banyuls-sur-Mer (P.-O.), région de collines boisées (Quercus suber) où abondent également Phlebotomus minutus (J.-A. Rioux et L. P. Knoepffler).

\section{$2^{\circ}$ Phototactisme.}

Grâce à la méthode des papiers huilés éclairés, nous avons pu mettre en évidence la nette attraction phototactique de Phlebotomus minutus + . Rappelons ici les résultats obtenus pour cette espèce au cours des campagnes 1965 et 1966.

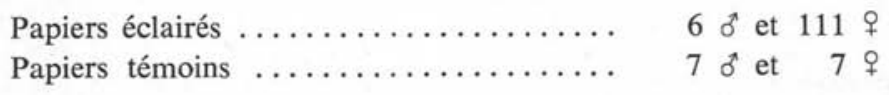

\section{$3^{\circ}$ Biotopes préférentiels.}

A l'état adulte, Phlebotomus minutus colonise les biotopes les plus variés. Toute- 
fois, on l'observe avec une particulière fréquence dans les lieux rocheux (falaises, ruines, murs de soutènement).

Dès 1963, les méthodes qualitatives de piégeages nous ont permis de mettre en évidence la grande abondance de cette espèce dans les cavités ménagées dans les murs de soutènement (barbacanes). En 1965, l'utilisation des méthodes quantitatives d'échantillonnages nous a permis de confirmer la grande richesse de ce biotope par rapport aux cavités rocheuses naturelles. Ainsi, pour un total de 15.304 Phlebotomus minutus capturés par la méthode des papiers huilés, le rendement moyen des barbacanes a atteint le chiffre élevé de 61,6 individus par mètre carré, contre 2,6 seulement pour les cavités naturelles.

Dans la barbacane, l'imago femelle de Phlebotomus minutus est d'ailleurs parfaitement à sa place car il y voisine avec le Lézard des murailles qui constitue son hôte préférentiel. Bien plus, étant donné sa richesse en matières organiques (déjections de reptiles et de micromammifères) et sa stabilité thermo-hygrométrique, ce type de milieu doit également convenir à la ponte et à l'évolution larvaire. Dans cette hypothèse on pourrait admettre que Phlebotomus minutus puisse effectuer la totalité de son cycle biologique dans cette niche et n'en sortir que pour sa dispersion.

PHLEBOTOMUS (PHLEBOTOMUS) PAPATASI (sCOPOLI, 1786)

Phlebotomus papatasi représente l'espèce «chef de file» de l'ancien sous-genre Phlebotomus s. st. Ce groupe, très homogène, ne comprend à l'heure actuelle que les trois espèces suivantes: Phlebotomus papatasi (Scopoli, 1786). Phlebotomus bergeroti (Parrot, 1934), Phlebotomus duboscqi (Neveu-Lemaire, 1908). Phlebotomus papatasi, très largement répandu d'Est en Ouest, occupe la zone d'influence mésogéenne ; Phlebotomus bergeroti couvre la bande désertique saharo-yéménite ; Phlebotomus duboscqi s'étend sur l'Afrique au Sud du Grand Désert. Il s'agit en somme de trois formes affines, véritables vicariantes géographiques, qui se succèdent du Nord au Sud, de la région méditerranéenne s.l. à la région éthiopienne.

Espèce type du genre, Phlebotomus papatasi est aussi le premier Phlébotome signalé en France (1909).

\section{Répartition géographique.}

La répartition française de Phlebotomus papatasi est très comparable à celle de Phiebotomus minutus. Comme lui il demeure inféodé aux départements méditérranéens sans pour autant se cantonner à l'ambiance littorale. (Gigondas: J. Coudert ; SaintJean-du-Gard: M. Sicart). En Italie, Phlebotomus papatasi atteint d'ailleurs les altitudes de $800 \mathrm{~m}$ (Gargano : A. Coradetti, G. Sacca et I. Neri) et $1.100 \mathrm{~m}$ (Sicile: S. Adler et O. Theodor). De même, sur les Hauts-Plateaux algériens, il dépasse en fréquence Phlebotomus perniciosus (Ed. Sergent). 


\section{Ethologie.}

Du point de vue trophique, Phlebotomus papatasi se présente comme une espèce essentiellement domestique et anthropophile, pénétrant dans les appartements et n'en sortant que pour pondre. Ainsi, en Macédoine, T. Simitch capture pendant la journée de 500 à 1.000 exemplaires dans les pièces habitées. En Yougoslavie, ce même auteur obtient un pourcentage variant entre 80 et $99 \%$ dans les habitations humaines contre $20 \%$ seulement dans les étables et les poulaillers. En France, malgré la rareté des observations on retrouve ce même comportement casanier. Ainsi à Marseille, J. Raynal et P. Le Gac ont capturé Phlebotomus papatasi sous moustiquaire. A. Montpellier, P. Mirouse (in M. Sicart, 1954) a récolté durant plusieurs années de nombreux exemplaires de cette espèce, dans son appartement urbain et plus précisément dans sa chambre à coucher. $\mathrm{Ce}$ comportement très particulier explique vraisemblablement la grande rareté de Phlebotomus papatasi dans notre statistique (1 q) portant cependant sur un total de 29.304 échantillons. Nous n'avons en effet qu'exceptionnellement chassé, de jour ou de nuit, dans les appartements habités.

PHLEBOTOMUS (PHLEBOTOMUS) MASCITTII GRASSI, 1908

\section{Répartition géographique.}

L'aire géographique actuelle de Phlebotomus mascittii s'étend au nord du Bassin méditérranéen, de la Grèce à la France. On ne le connaît ni d'Espagne, ni d'Afrique du Nord. En France la dissémination des localités à l'ensemble du territoire plaide en faveur d'une répartition nettement septentrionale. Nul doute qu'un inventaire plus approfondi ne fasse découvrir cette espèce dans les pays frontaliers, tels l'Allemagne, le Luxembourg ou la Belgique (1).

Au cours de ce travail, nous avons observé Phlebotomus mascittii à des altitudes très diverses depuis la région côtière (Toulon) jusqu'aux lisières des Chênaies caducifoliées. Dans le Gard, nos captures l'indiquent à $600 \mathrm{~m}$ (Esparon); dans l'Hérault, il atteint $626 \mathrm{~m}$ à Saint-Pierre-de-la-Fage. Toutefois, le nombre très faible de nos récoltes enlève à ces altitudes limites une grande partie de leur valeur. Il est vraisemblable que cette espèce, qui atteint en France les régions du Nord et de l'Est, parvient sans difficultés à l'étage du Hêtre, tout au moins dans le «Midi » méditerranéen.

\section{Ethologie.}

Malgré le nombre de localités inventoriées, l'éthologie de cette espèce est encore mal connue. Son anthropophilie ne fait cependant aucun doute ainsi qu'en témoignent les publications de M. C. et J. Colas-Belcour (1929), de G. Lavier et A. Ristorcelli (1929-1941), de J. Callot (1950) et de C. Toumanoff et R. Chassignet (1954). Dans cette

(1) Phlebotomus mascittii est déjà connu en Suisse (H. Gashen, 1945). 
dernière observation, assez exceptionnelle, les auteurs signalent même une très forte agressivité, manifestée en plein jour, dans un tunnel désaffecté des environs de PortoVecchio (Corse). L'activité diurne de cette espèce a d'ailleurs été signalée par plusieurs auteurs dont récemment M. Sicart (1951).

Pour notre part, nous ne pouvons apporter aucune contribution de valeur à la connaissance écologique de Phlebotomus mascittii car en 5 ans de capture, nous ne l'avons identifié que 27 fois $\left(19 q\right.$ et $\left.8 \sigma^{\star}\right)$. Parmi les 19 femelles du lot, 8 provenaient de chasses nocturnes à la lampe, 2 de chasses diurnes dans les fissures de murs et de rochers et 9 de chasses aux papiers huilés ; aucune n'était capturée sur appât humain.

\section{PHLEBOTOMUS (PHLEBOTOMUS) PERNICIOSUS NeWSTEAD, 1911}

\section{Répartition géographique.}

Décrit de l'Ile de Malte par R. Newstead en 1911, Phlebotomus perniciosus occupe un très vaste territoire géographique englobant les régions médio-européennes et méditerranéennes jusqu'au Sahara.

La répartition française de Phlebotomus perniciosus est assez comparable à celle de Phlebotomus mascittii. Comme ce dernier, il doit certainement exister dans les départements du Nord et de l'Est où aucune station n'est connue jusqu'à présent (11).

\section{TABleau III}

\section{ALTITUDES MAXIMALES DES STATIONS POUR :}

Phlebotomus ariasi

Phlebotomus minutus

Phlebotomus perniciosus

Sud de la France

(captures de 1963 à 1965)

\begin{tabular}{|l|c|c|c|c|c|}
\hline & $\begin{array}{c}\text { PYRENEES } \\
\text { orientales }\end{array}$ & ESPINOUSE & AIGOUAL & LOZERE & ALPES \\
\hline Phlebotomus ariasi & $1415 \mathrm{~m}$. & $620 \mathrm{~m}$. & $920 \mathrm{~m}$. & $820 \mathrm{~m}$. & $1100 \mathrm{~m}$. \\
\hline Phlebotomus minutus & $747 \mathrm{~m}$. & $620 \mathrm{~m}$. & $850 \mathrm{~m}$. & $730 \mathrm{~m}$. & $641 \mathrm{~m}$. \\
\hline Phlebotomus perniciosus & $640 \mathrm{~m}$. & $440 \mathrm{~m}$. & $600 \mathrm{~m}$. & & \\
\hline
\end{tabular}

(11) Phlebotomus perniciosus est en effet connu de l'lle de Jersey (P.-J. Marett, 1923). Annales de Parasitologie humaine et comparée (Paris), t. 42, 1967, nº 6 
Paradoxalement, pour une espèce à vaste répartition, Phlebotomus perniciosus ne semble pas s'élever en altitude, tout au moins dans nos régions. Ainsi, dans le Massif du Caroux, nos captures le signalent jusqu'à $440 \mathrm{~m}$ seulement, alors que Phlebotomus ariasi et Phlebotomus minutus atteignent $620 \mathrm{~m}$ (12). Dans le massif de l'Aigoual, il ne dépasse pas $600 \mathrm{~m}$ contre $850 \mathrm{~m}$ pour Phlebotomus minutus et $920 \mathrm{~m}$ pour Phlebotomus ariasi. Dans les Pyrénées-Orientales, il n'est plus observé au-delà de $640 \mathrm{~m}$, alors que Phlebotomus minutus remonte à $747 \mathrm{~m}$ et Phlebotomus ariasi à $1.415 \mathrm{~m}$ (tabl. III).

\section{Ethologie.}

L'éthologie de Phlebotomus perniciosus a fait l'objet de nombreuses publications tant en Europe qu'en Afrique du Nord. La plupart d'entr'elles insistent sur trois points essentiels : l'endophilie, la zoophilie et l'évolution diphasique.

$1^{\circ}$ L'endophilie, propriété fondamentale de cette espèce, est illustrée par les captures de nombreux auteurs. Ainsi, à Marseille, J. Raynal et P. Le Gac obtiennent un fort pourcentage de Phlebotomus perniciosus à l'intérieur des maisons d'habitations, des écuries et des clapiers (1933).

Notre expérience confirme indirectement ces observations : en 27 séances de chasse nocturne à l'extérieur, sur appât humain, nous n'avons capturé que trois exemplaires de Phlebotomus perniciosus. Moins sélective, la chasse nocturne à la lampe donne cependant des résultats dans l'ensemble assez comparables (tabl. I.).

Toutefois, au cours de la journée, Phlebotomus perniciosus semble « gîter habituellement hors des habitations humaines 》 (J. Raynal et P. Le Gac, 1933) ; ce qui revient à dire que son comportement endophile s'exprime surtout à l'occasion du repas sanguin, à l'inverse de Phlebotomus papatasi dont les lieux de piqûre sont aussi les lieux de repos.

$2^{\circ}$ La zoophilie, autre caractère de Phlebotomus perniciosus, se manifeste à l'égard de nombreux mammifères, en particulier du Chien qui, d'après J. Parrot et A. Donatien, semble être préféré à l'homme.

En ce qui concerne le « Midi » méditerranéen, nos captures de Phlebotomus perniciosus sont trop peu nombreuses pour permettre une interprétation correcte des faits. Toutefois, il est important de souligner sa très faible attirance pour l'Homme ou le Chien. Ainsi, au cours de nos chasses noctures à l'extérieur et à l'intérieur des habitations nous n'avons que rarement capturé cette espèce contrairement à Phlebotomus ariasi.

$3^{\circ}$ L'évolution diphasique, signalée par certains auteurs en Sicile et en Algérie, correspondrait à deux vagues d'éclosion. La première, vernale, relativement discrète,

(12) Ces altitudes n'ont évidemment aucun caractère absolu. Elles indiquent simplement le décalage relatif des trois espèces. La limite altitudinale des trois espèces étudiées est nettement plus basse dans les massifs peu élevés (Espinouse, Aigoual). Ici, comme pour la flore, intervient l'effet de crête. 
serait formée par des imagos provenant des pontes d'automne, c'est-à-dire des larves ou des nymphes ayant passé l'hiver en diapause. La vague d'automne, beaucoup plus importante, proviendrait des larves issues de la génération de printemps. L'intervalle entre les deux vagues représenterait assez fidèlement le temps d'évolution de la génération d'été, temps d'ailleurs établi expérimentalement par L. Parrot, A. Donatien et F. Lestoquard en 1933 (134 à 216 jours à $\left.22^{\circ}-24^{\circ}\right)$.

Or, sans qu'il soit possible d'en donner une explication rationnelle, dans le sud de la France, Phlebotomus perniciosus n'évolue pas suivant un tel schéma. La méthode des papiers adhésifs (figs. 13 et 14), ne montre en effet qu'un seul maximum situé au cœur de la saison chaude (15 juillet-15 août).

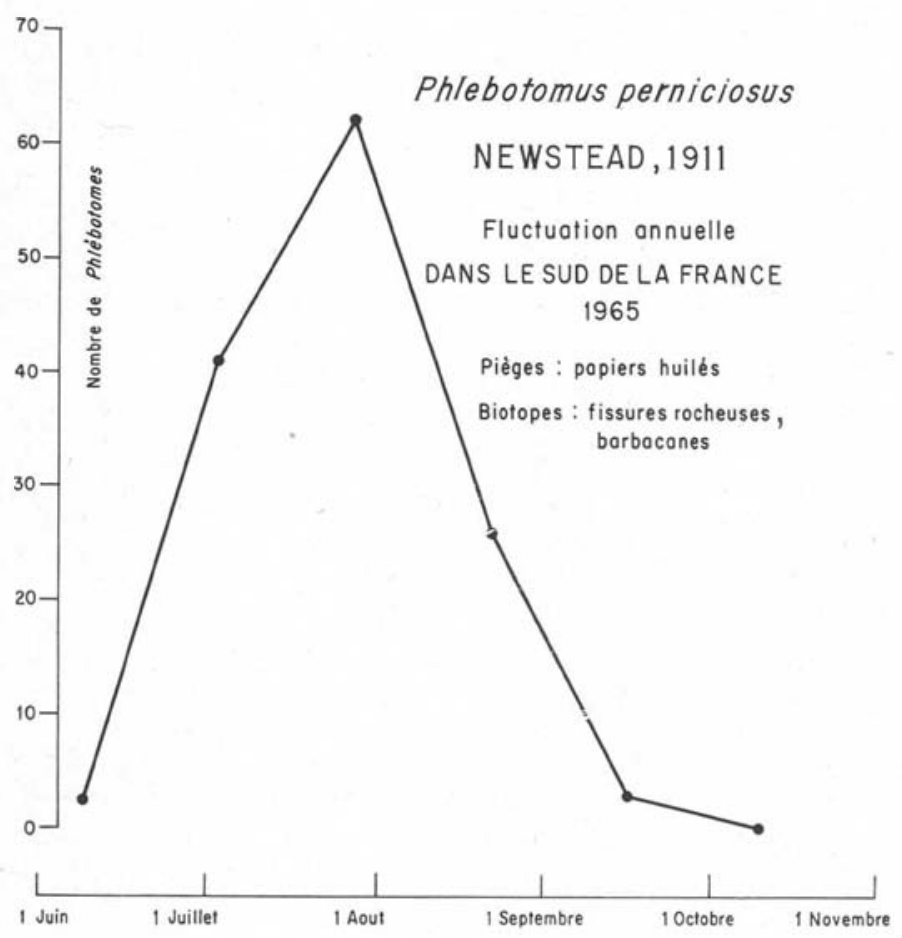

FIG. 13

Quant à la durée totale de la période d'activité imaginale, elle ne peut être indiquée avec précision en raison du nombre trop faible des captures. Mentionnons cependant qu'elle s'étend au moins de juin à septembre, ainsi qu'en témoignent les résultats des piégeages systématiques effectués en 1965 dans les massifs Lozère-Cévennes-MontagneNoire-Espinouse. 
PHLEBOTOMUS (PHLEBOTOMUS) ARIASI TONNOIR, 1921

\section{Répartition géographique.}

En France Phlebotomus ariasi s'étend sur l'ensemble des départements méditerranéens et remonte de part et d'autre du Massif Central ; en particulier à l'Ouest où il atteint la Vienne et la Sarthe (13).

Phlebotomus ariasi s'élève en altitude jusqu'à l'étage du Hêtre. Il dépasse donc largement les autres espèces en particulier Phlebotomus perniciosus. Ainsi, en 1965, nous l'avons capturé à $920 \mathrm{~m}$ dans le Massif de l'Aigoual, à $850 \mathrm{~m}$ dans le Massif du Lozère et à $1.100 \mathrm{~m}$ dans les Basses-Alpes. Dans les Pyrénées-Orientales, cette même espèce atteint l'altitude record de $1.415 \mathrm{~m}$ à Mont-Louis (tabl. III).

\section{Ethologie.}

\section{$1^{\circ}$ Cycle annuel et journalier.}

Dans le sud de la France, la période d'activité imaginale de Phlebotomus ariasi semble beaucoup plus étalée qu'on ne pouvait le supposer.

Ainsi, le 29 septembre 1963, un exemplaire mâle a pu être capturé dans une barbacane à Villeneuvette (Hérault). En 1964, nos premières captures d'adultes ont été réalisées le 11 juin (Villeneuvette : 4 + ), les dernières, exactement trois mois plus tard, le 11 septembre (Montdardier: $8 \delta^{\star}$ ). Bien plus, la même année, dans un lot de 13 exemplaires récoltés le 27 juin dans une cabane de cantonnier au col de Fourtou (P.-O.), à $800 \mathrm{~m}$ d'altitude, une femelle gravide renfermait déjà des œufs en état d'évolution avancée. En 1965, les papiers huilés placés le 5 novembre et relevés le 25 du même mois ont capturé 12 Phlebotomus ariasi sur le flanc Sud du Caroux (Hérault) entre 260 et $350 \mathrm{~m}$. Récemment, le 27 mai 1967, une femelle était capturée dans une pièce habitée à Villeneuvette (Hérault).

On peut donc raisonnablement penser que les premiers adultes s'envolent au début de mai, les derniers prolongeant leur activité jusqu'en novembre ; ce qui revient à fixer à six et peut-être à sept mois la durée de la période imaginale (14).

Plus difficile s'avère l'étude du rythme d'éclosion des imagos au cours de cette période d'activité. En l'occurrence, les méthodes manuelles d'échantillonnage telles que la capture nocturne contre les murs ou diurne dans les cavités, ne sont d'aucune utilité en raison de nombreuses causes d'erreurs qu'elles comportent (coefficient personnel

(13) En dehors de la France, cette espèce est connue d'Espagne (A. Tonnoir, 1921), d'Italie (J.-A. Rioux, M. Coluzzi, O. Bain et J.-P. Baudouy, 1964), d'Algérie (L. Parror, 1936), du Maroc (J. Gaud, 1945) et de Tunisie (H. Croset, J.-A. Rioux et B. Juminer, 1966).

(14) Comme pour Phlebotomus perniciosus, la durée du cycle larvaire paraît extrêmement longue. Ainsi trois pontes obtenues le 22 août 1965 à partir de Phlebotomus ariasi capturés le 17 août au Villaret $(270 \mathrm{~m}$, Gard), ont éclos le 30 et 31 août. Le 5 octobre seulement les larves parvenaient au IV $^{\circ}$ stade (température d'élevage $25^{\circ}$ ). 
des piégeurs, durée du piégeage, influence météorologique). En définitive, elles expriment plus les fluctuations des « sorties * journalières que celles des éclosions proprement dites.

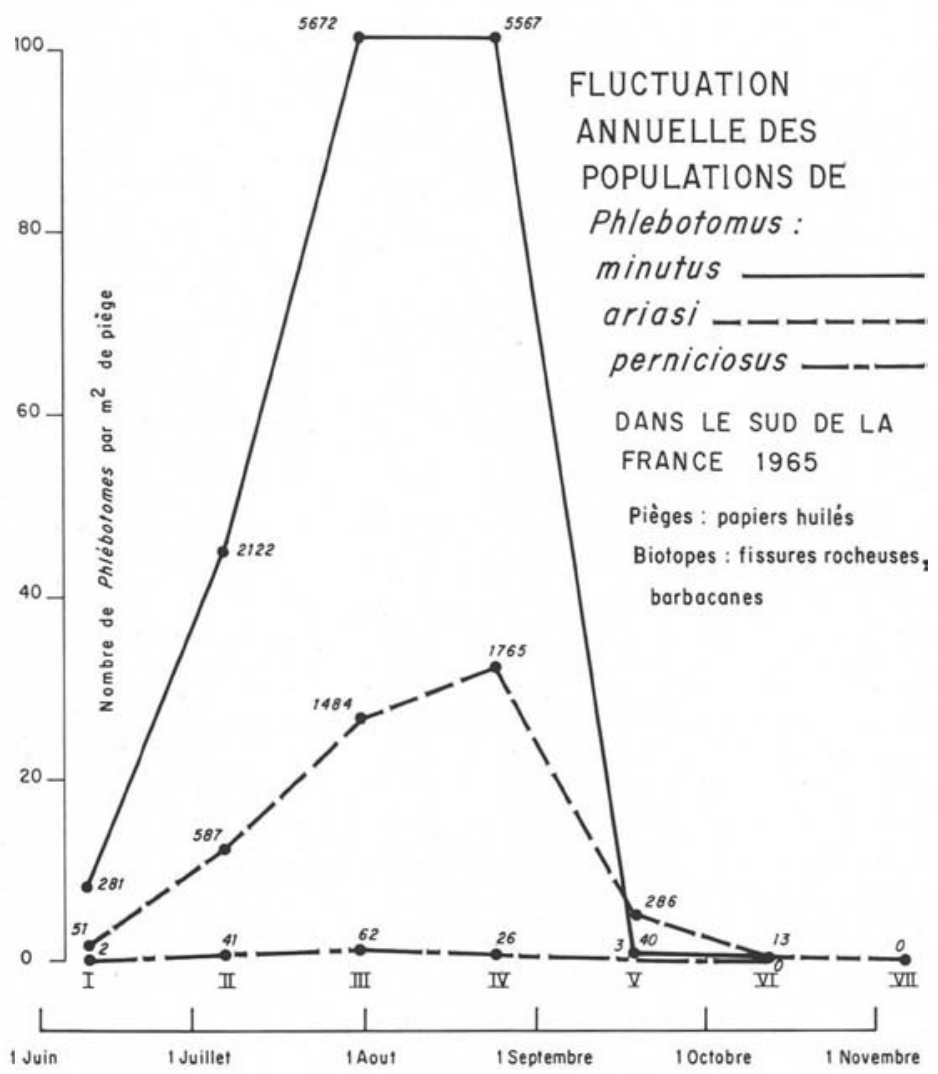

FIG. 14

Par contre, la technique des papiers huilés laissés à demeure dans les gîtes de repos constitue, dans le cas particulier, un excellent procédé d'échantillonnage. La continuité et la durée du piégeage d'une part, les caractères écologiques particuliers des stations piégées (cavités abritées, habitations) d'autre part, permettent d'atténuer, voire de supprimer les fluctuations (15). Le dépouillement des divers transects réalisés en

(15) En 1966, à l'occasion d'un séjour à La Borie-Nouvelle (Hérault, alt. $500 \mathrm{~m}$ ), nous avons pu démontrer l'influence des facteurs météorologiques sur les «sorties» des Phlébotomes :

«Du 8 au 20 juillet 1966, nous disposons un piège lumineux standard (six papiers huilés $n^{\circ} 4$ ) contre un mur de pierres sèches. Chaque soir, le dispositif est mis en marche à $20 \mathrm{~h} 30$ et arrêté à $3 \mathrm{~h}$. Les papiers sont retirés le lendemain vers $10 \mathrm{~h}$ et dépouillés extemporanément.

Les résultats, illustrés par les figures 10 et 15 , montrent une corrélation nette entre les 


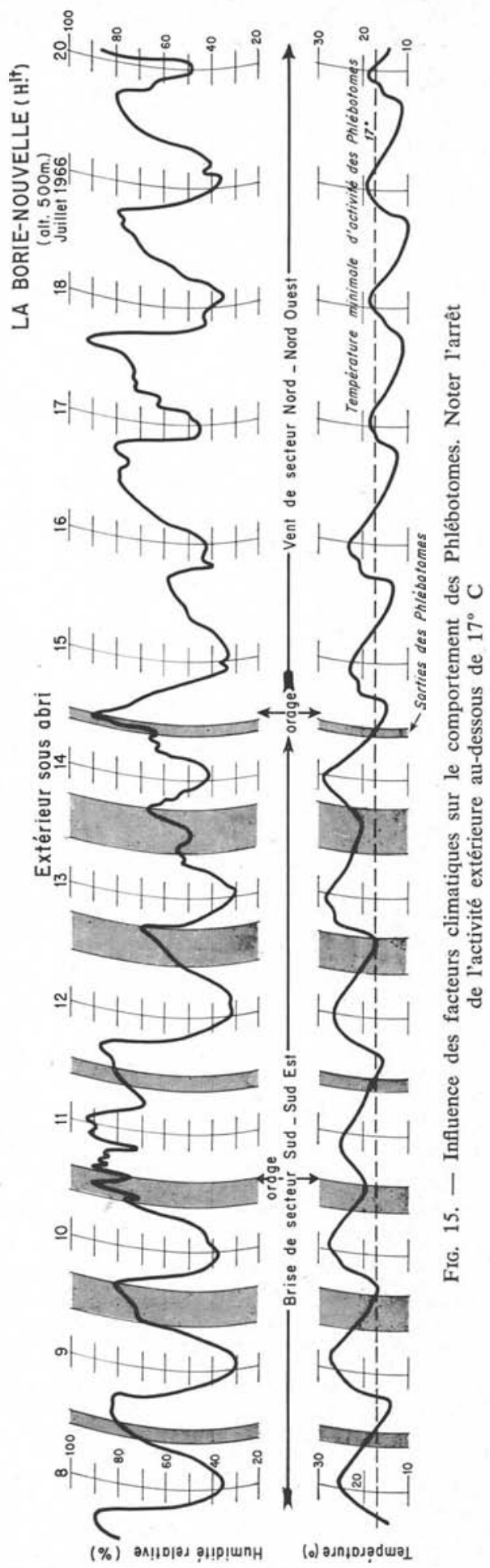


1965 a d'ailleurs permis de tracer une série de courbes correspondant à l'acmé de la période chaude (fig. 14).

\section{$2^{\circ}$ Accouplement.}

Au cours de nos chasses nocturnes nous avons capturé à plusieurs reprises des copula posées sur les murs. Toutefois en aucun moment nous n'avons observé les temps successifs de l'accouplement tels que les a décrit avec précision A. Baudrimont dans son mémoire de 1946 (16).

\section{$3^{\circ}$ Comportement trophique.}

1. L'anthropophilie de Phlebotomus ariasi est amplement démontrée par les résultats de nos chasses nocturnes, méthode où seuls sont capturés les individus piquant ou s'apprêtant à piquer l'Homme, à l'exception de ceux simplement attirés par la lumière (tabl. I). Ainsi sur 478 Phlébotomes capturés de la sorte durant les étés 1961 à 1965 , on dénombre :

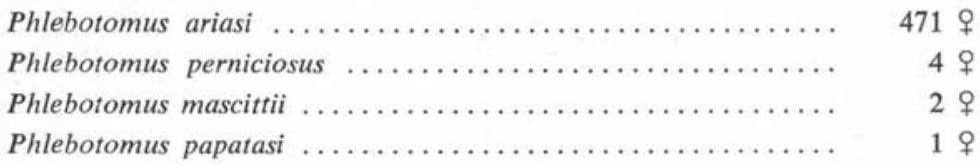

Au demeurant, Phlebotomus ariasi se gorge également sur les animaux domestiques ou sauvages. Ainsi, dans les Cévennes, les clapiers sont assez souvent envahis. D'ailleurs en laboratoire, nous avons pu obtenir la réplétion sanguine sur Lapins et Cobayes.

2. L'exophagie représente, au même titre que l'anthropophilie, le trait éthologique fondamental de Phlebotomus ariasi. Ce caractère s'exprime de manière particulièrement nette dans le tableau récapitulatif où le chiffre des captures réalisées à l'extérieur (sur appâts et contre les murs) est environ 5 fois plus élevé (2.044 \$) que celui des captures effectuées à l'intérieur (404 \$).

Toutefois, s'il est aisé de mettre en évidence cette endophilie relative, plus difficile est d'en préciser le mécanisme. L'hypothèse d'une fraction endophile était certes sédui-

fluctuations des captures et les variations de conditions météorologiques (températures, précipitations, vents).

De même, grâce à ces données, nous avons pu fixer à $17^{\circ} \mathrm{C}$ la température minimale d'activité de Phlebotomus ariasi. Le graphique de la figure 15 correspondant à la courbe des températures et des humidités relatives (extérieur sous abri) rend compte de la durée approximative de cette activité. Il explique en particulier l'intensité et l'étalement des "vagues de pullulation" observées les 9,12 et 13 juillet $1966 . »$

(16) \& Une femelle (Phlebotomus ariasi) venant de se poser sur l'abat-jour de ma lampe, attire bien vite mon attention par de rapides et très minimes tremblements des ailes. Un mâle survient, se place tout à côté et présente lui aussi de minuscules soubresauts, mais de tout le corps. Il s'approche, la femelle s'éloigne de trois ou quatre centimètres et se pose aussitôt, toujours en trémulant des ailes. Le mâle s'avance à nouveau, sans interrompre lui non plus ses petits sursauts généralisés. Tout à coup, il s'élance et, se retournant tout d'une pièce, sans une hésitation, saisit brutalement l'extrémité postérieure de la femelle dans sa double pince génitale où elle est solidement maintenue par les fortes et longues épines des gonapophyses supérieures. » (A. Baudrimont, 1946). 
sante, mais difficile à démontrer, car relevant des méthodes de la génétique écologique. Aussi nous sommes-nous attachés à l'étude de facteurs élémentaires qui rendent compte tout au moins partiellement, de ce comportement: la sensibilité au vent et au froid d'une part, l'attraction lumineuse d'autre part :

a) Sensibilité anémo-thermique: le facteur thermique intervient de façon non négligeable, tout au moins pour démasquer cette fraction endophile. Ainsi, en début et fin de saison chaude il est possible de capturer des femelles en activité dans les habitations alors que dans le même temps aucun adulte ne vole au-dehors.

Cette véritable « inversion » peut d'ailleurs s'observer en plein été à l'occasion d'un refroidissement ou d'un orage :

Du 8 au 20 juillet 1966, à La Borie-Nouvelle (Hérault, alt. $500 \mathrm{~m}$ ) nous mettons en batterie deux pièges lumineux «standard \$ $\left(6\right.$ papiers huilés $\mathrm{n}^{\circ} 4$ par piège) l'un à l'extérieur, contre un mur, l'autre à l'intérieur dans une soupente. Le soir du 14 juillet, après un violent orage, la température accuse un net fléchissement, cependant que la brise de secteur Sud cède la place au mistral. A partir de cette date, aucun Phlébotome n'est plus observé au dehors (fig. 10 et 15). Le dépouillement des pièges réalisés en fin de campagne confirme l'observation (12 nuits de piégeage): Phlebotomus ariasi prédomine à l'intérieur (tabl. IV).

TABleau IV

\begin{tabular}{|c|c|c|c|c|c|c|}
\hline & & \multicolumn{2}{|c|}{ Piège extérieur } & \multicolumn{3}{|c|}{ Piège intérieur } \\
\hline \multirow{3}{*}{ 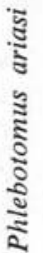 } & Mâles & 44 & $33,6 \%$ & 87 & 66,4 & \\
\hline & Femelles & 33 & $34,3 \%$ & 63 & 65,6 & $\%$ \\
\hline & ot et $q$ & 77 & $34 \%$ & 150 & 66 & $\%$ \\
\hline
\end{tabular}

b) Attraction phototactique: le phototactisme de Phlebotomus ariasi sera étudié au chapitre suivant. Contentons-nous de souligner ici le rôle de l'attraction lumineuse dans la pénétration de cette espèce à l'intérieur des habitations.

Quoi qu'il en soit, la présence de Phlebotomus ariasi dans les maisons, pour si discrète qu'elle soit, n'en pose pas moins l'important problème de son intervention dans le cycle du Kala-Azar en pays cévenol. Bien plus, dans cette éventualité, la rareté du vecteur dans les habitations expliquerait la faible incidence de la Leishmaniose viscérale humaine par rapport à la Leishmaniose canine dont la transmission extra muros serait dès lors le « mode habituel » épidémiologique.

Pour revenir à l'exophilie de Phlebotomus ariasi, il est capital de souligner que ce comportement se manifeste non seulement aux environs immédiats des fermes et des villages, mais également loin de toute habitation, en « pleine nature ». 


\section{$4^{\circ}$ Phototactisme.}

Dans un article sur l'éthologie des Phlebotomus perniciosus de R. M. Nicoli et J. Nicoli (1963) insistent avec à propos sur les incertitudes, voire les contradictions relevées dans la littérature au sujet des réactions phototactiques des Phlébotomes.

$\mathrm{Au}$ cours de cette enquête nous avons tenté à notre tour une approche quantitative du problème, et ce, dans le double but de faciliter l'échantillonnage des populations locales (mise au point d'un piège lumineux) et d'expliquer pro parte la pénétration de certaines espèces dans les maisons habitées.

Pour ce faire nous avons mis à l'essai plusieurs montages comportant des sources lumineuses différentes, soit par l'intensité, soit par la longueur d'ondes. Ces montages nous ont permis de démontrer (17) :

1. Que Phlebotomus ariasi est nettement attiré par la lumière (tabl. V).

2. Que les femelles de cette espèce sont plus attirées que les mâles (résultats statistiquement significatifs).

\section{Tableau V. - Etude du phototactisme des Phlébotomes par la méthode des papiers huilés éclairés (Captures 1965-1966)}

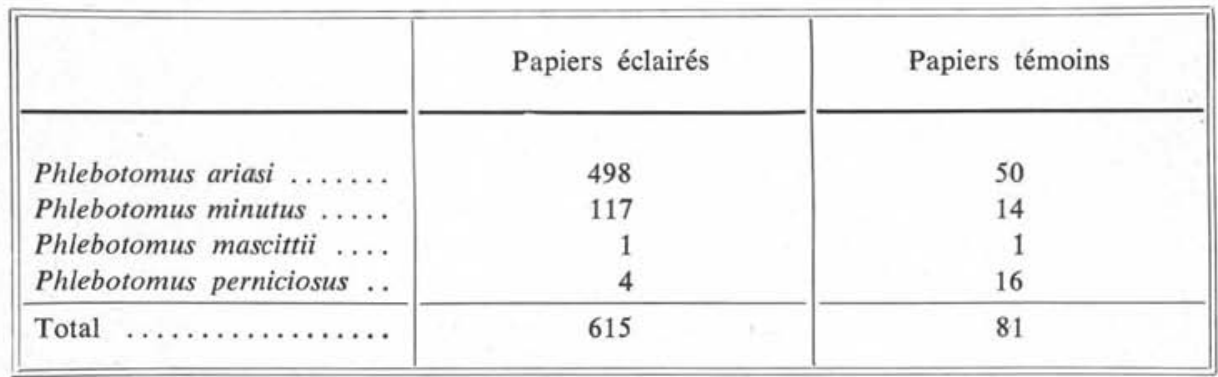

En fait, avec l'expérience précédente, nous abordons un autre aspect du phototactisme sur lequel insistent R. M. Nicoli et J. Nicoli : la réponse à l'albédo du support. Ces auteurs signalent en particulier la répulsion de Phlebotomus perniciosus pour les supports de couleur claire. Leurs observations faites sur des surfaces d'essai de tonalités différentes sont très démonstratives à cet égard, les $9 / 10^{\circ}$ des Phlébotomes recueillis l'étant sur des surfaces d'albédo faible (15 à $10 \%)$.

Ces conclusions, applicables à Phlebotomus ariasi, expliquent vraisemblablement le comportement paradoxal de cette espèce vis-à-vis de la lumière, comportement que nous

(17) La variante $\mathrm{C}$ du piège lumineux tel que nous l'avons décrit plus haut (p. 575) a permis une interprétation statistique suffisante des résultats. Ainsi, sur 255 Phlebotomus ariasi capturés par cette méthode, soit :

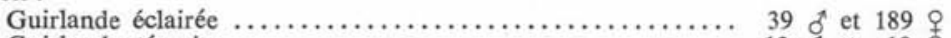

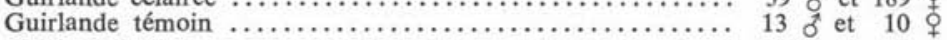

le calcul de l'écart réduit par rapport à l'hypothèse $50 \%$ a montré une différence hautement significative aussi bien pour des femelles $(94,9 \%$ sur la guirlande éclairée $\varepsilon=12,2)$ que pour les mâles ( $72,2 \%$ sur la guirlande éclairée $\varepsilon=3,26)$. 
avons observé, dans de nombreuses stations du « Midi » méditerranéen. Ainsi, lors de nos chasses nocturnes, à la lampe de poche, nous avons toujours été frappés par la répartition des Phlébotomes par rapport au faisceau lumineux, la plupart se posant non pas dans la partie centrale la plus éclairée, mais en bordure, dans la frange de pénombre.

Quoi qu'il en soit, la présence de Phlebotomus ariasi à l'intérieur des maisons trouverait en partie son explication dans un simple phénomène d'attraction phototactique. Dès lors cette espèce pourrait se comporter comme un véritable « vecteur de liaison ", susceptible, en d'autres termes de servir d'intermédiaire entre les réservoirs de virus extérieurs (Chien et Renard) et l'enfant, habituellement piqué à l'intérieur.

\section{ÉTUDE SYNTHÉTIQUE. COROLLAIRES ÉPIDÉMIOLOGIQUES}

Parmi les trois principales espèces anthropophiles présentes dans le sud de la France, Phlebotomus ariasi a surtout retenu notre attention comme «vecteur habituel » des Leishmanioses. En faveur de cette hypothèse, les arguments indirects sont nombreux; dans le chapitre analytique nous les avons discutés à plusieurs reprises. Pour plus de clarté nous les reprendrons en les résumant sous trois chefs, à savoir, l'abondance absolue de l'espèce sur l'ensemble de la zone prospectée, son maximum de densité dans l'aire d'endémie leishmanienne, ses particularités écologiques et éthologiques qui limpliquent aussi bien dans la transmission des Leishmanioses animales qu'humaines. Nous discuterons enfin des arguments épidémiologiques directs que nous avons pu développer récemment.

\section{I. - Arguments indirects}

$1^{\circ}$ Dans le « Midi 》 méditerranéen, Phlebotomus ariasi représente l'espèce anthropophile dominante.

Exception faite de l'herpétophile Phlebotomus minutus, Phlebotomus ariasi représente effectivement l'espèce dominante.

Ainsi, sur 12.772 Phlébotomes groupant la masse totale des espèces anthropophiles identifiée de 1961 à 1966, on dénombre :

Phlebotomus ariasi ..........

Phlebotomus perniciosus .....

Phlebotomus mascittii ........

Phlebotomus papatasi ........
12.312 exemplaires $(96,399 \%)$

432 exemplaires $(3,383 \%)$

27 exemplaires $(0,211 \%)$

1 exemplaire $(0,007 \%)$

Si l'on considère à présent les proportions ariasi/perniciosus par mode de piégeage, on trouve toujours un chiffre supérieur à $94 \%$ (tabl. I). Cette extrême abondance de Phlebotomus ariasi semble avoir été méconnue jusqu'à ce jour. Seule l'observation du 
Portugais J. Ferreirares (1956) en fait mention. Dans la vallée du Douro, cet auteur a pu récolter au cours d'un cycle annuel, 2.665 Phlebotomus ariasi contre 3.087 Phlebotomus perniciosus.

$2^{\circ}$ Dans l'aire d'endémie leishmanienne, Phlebotomus ariasi présente sa densité maximale.

Comme pour la plupart des insectes vulnérants, autrement difficile s'avère l'expression exacte de l'abondance relative des espèces selon les milieux et les étages

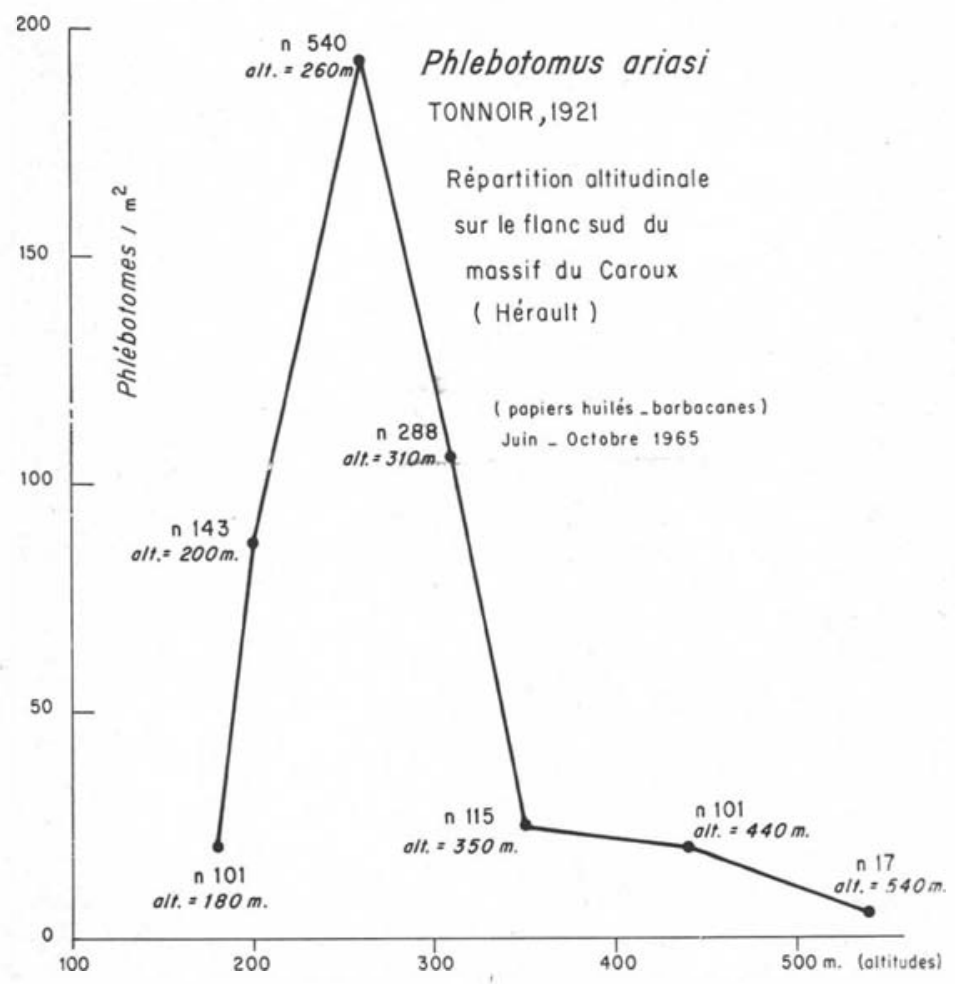

Fig. 16

altitudinaux. Les procédés manuels de captures, trop entachés par le coefficient personnel des piégeurs, ne permettent pas un échantillonnage rationnel. Par contre, la méthode des papiers huilés correspond parfaitement aux nécessités techniques de cette étude. Les pièges, mis en place dans les mêmes stations et récupérés à intervalles fixes (3 semaines), permettent en effet d'exploiter simultanément l'ensemble d'une région, des zones basses aux culmens. 
L'expression de la densité relative est alors possible en ramenant, pour chaque espèce, le nombre d'individus capturés au $\mathrm{m}^{2}$ de piège. En échantillonnant régulièrement les mêmes stations pendant les mois d'activité imaginale, on peut ainsi obtenir des chiflres possédant une réelle valeur statistique.

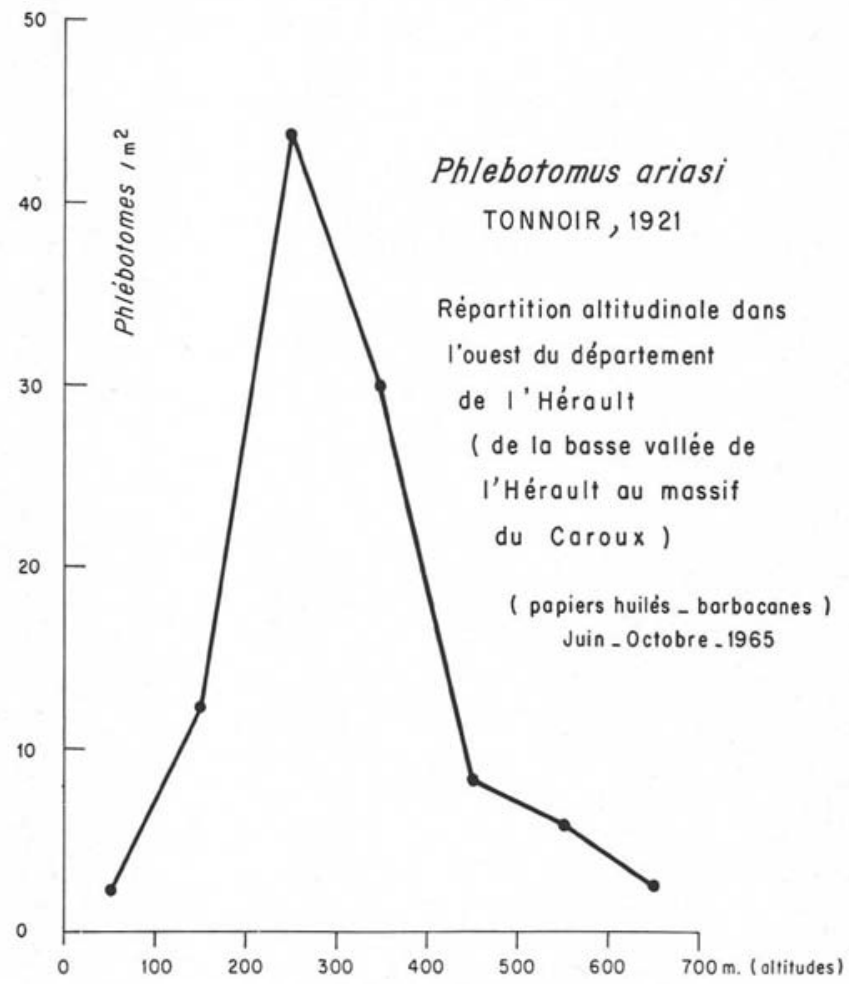

FIG. 17

C'est précisément pour réaliser un tel programme que nous avons mis au point et exploité les «itinéraires-transects », dont nous avons fait état précédemment. Rappelons simplement que ces itinéraires intéressent les principaux étages de végétation (climax climaciques) depuis les basses plaines littorales (Languedoc, Camargue), jusqu'aux sommets des massifs Espinouse-Montagne Noire-Cévennes.

Pour Phlebotomus ariasi les résultats, exprimés par étages de végétation, montrent effectivement un maximum de densité dans l'aire d'endémie leishmanienne (fig. 16, 17 et 18 et tabl. IV). Dans cette zone qui correspond à l'étage collinéen de la Chênaie d'Yeuse montagnarde et de la Chênaie mixte (fig. 19 et 20), cette espèce atteint le chiffre élevé de $26,75 / \mathrm{m}^{2}(61 \%)$, alors que dans la Chênaie d'Yeuse de plaine, la Chênaie caducifoliée et la Hêtraie, ce chiffre n'est que de $4,98 / \mathrm{m}^{2}(14 \%), 16,93 / \mathrm{m}^{2}$ $(24 \%)$ et $0,55(1 \%)$. 
TABleau VI. - Fréquence des trois principales espèces de Phlébotomes aux divers étages de végétation en Provence - Languedoc

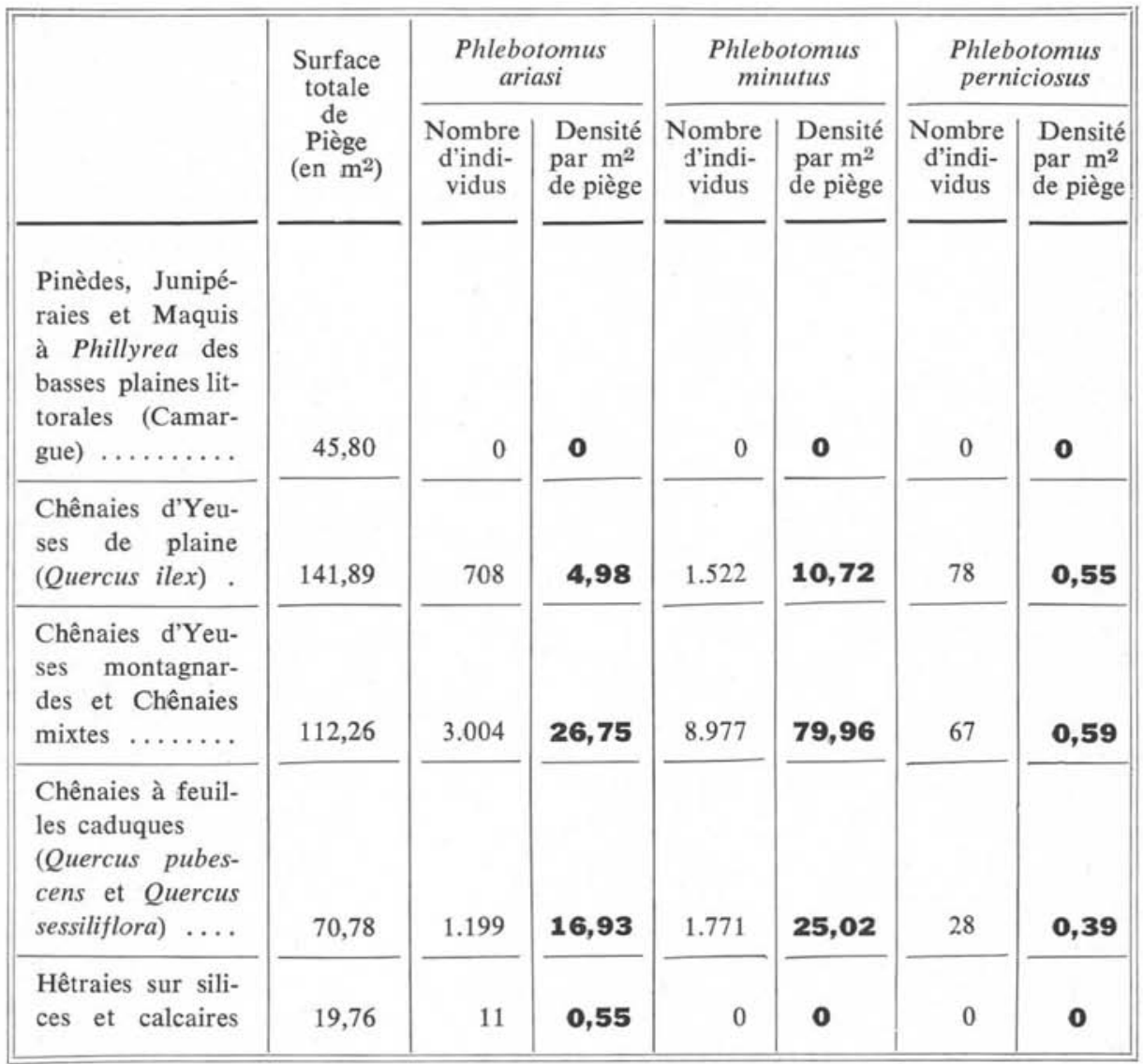

Ainsi la corrélation constatée entre l'étage de densité maximale des Phlébotomes et la zone d'endémie leishmanienne démontre, si besoin était, le rôle prépondérant joué par ces Diptères dans le « mode habituel » épidémiologique.

A l'opposé, la Tique du Chien (Rhipicephalus sanguineus), très abondante sur les côtes sableuses (Camargue en particulier), ne peut intervenir qu'accidentellement dans le processus de transmission (18). En aucune manière elle ne saurait entretenir, à elle seule, le « foyer naturel » du sud de la France.

(18) Rappelons que le rôle de Rhipicephalus sanguineus dans la transmission des Leishmanies, évoqué par G. Blanc et J. Caminopetros (1930 et 1937), n'a pu être confirmé. 
$3^{\circ}$ Le comportement de Phlebotomus ariasi lui confère un rôle prépondérant dans le cycle épidémiologique des Leishmanioses.

L'éthologie de Phlebotomus ariasi apporte enfin une autre série d'arguments non moins importants. Ainsi qu'il a été précédemment développé, Phlebotomus ariasi se comporte en effet comme espèce ubiquiste, à la fois sauvage et domestique, endo-exo-

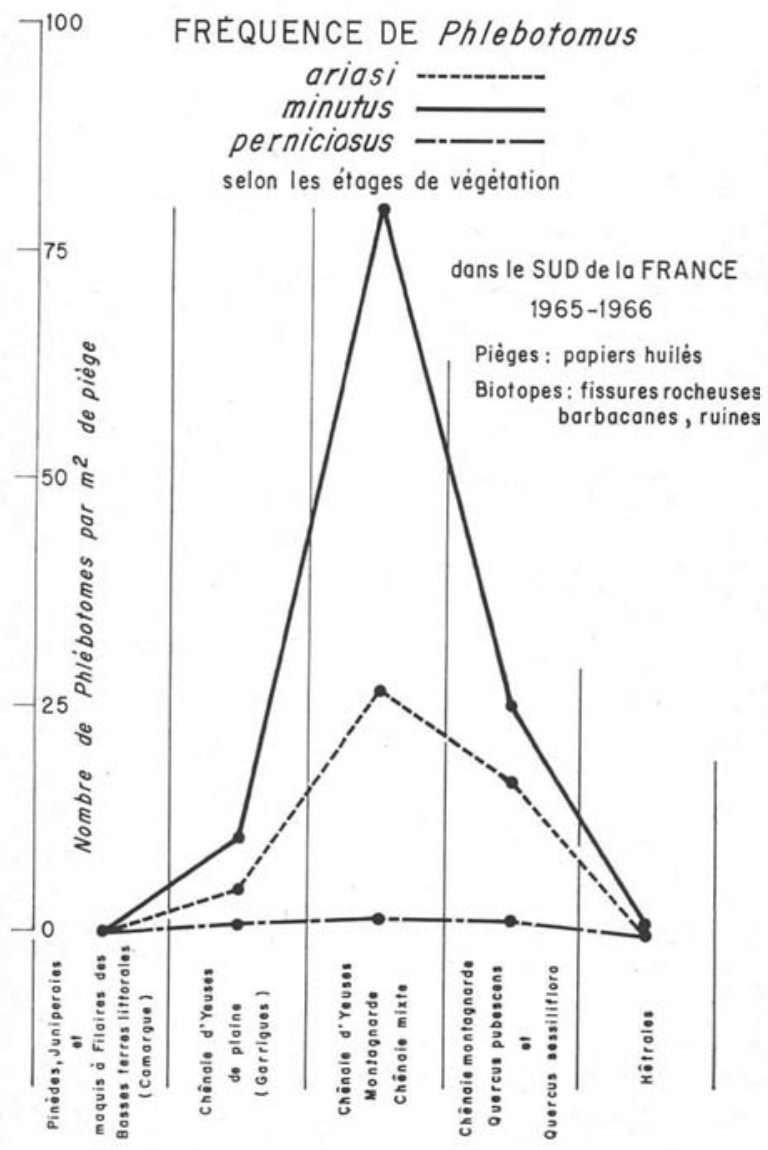

FIG. 18

phile et anthropo-zoophile, donc susceptible de servir de trait d'union entre les réservoirs de virus sauvages et domestiques d'une part, entre ces réservoirs et l'Homme d'autre part. Bien plus, il manifeste une attraction majeure pour l'Homme ainsi qu'en témoignent des résultats de captures nocturnes sur appâts humains (tabl. I et VII). La transmission à l'Homme serait donc essentiellement assurée par cette espèce. 


\begin{tabular}{|c|c|c|c|c|c|}
\hline & $\begin{array}{l}\text { Phle } \\
\text { pern }\end{array}$ & $\begin{array}{l}\text { mus } \\
\text { ius }\end{array}$ & $\begin{array}{r}\text { Phle } \\
a\end{array}$ & mus & \\
\hline & Q & $\sigma^{\prime \prime}$ & q & $\sigma^{\prime \prime}$ & $\frac{A \&}{A \&+P} \%$ \\
\hline $\begin{array}{c}\text { Roquedur-le-Bas } \\
\text { (Gard) } \\
14-\mathrm{VII}-62 \\
\text { no11 }\end{array}$ & 2 & 0 & 83 & 15 & $97,6 \%$ \\
\hline $\begin{array}{c}\text { Cessenon } \\
\text { (Heroult) } \\
19-\text { VII }-62 \\
n^{\circ} 12 \\
\end{array}$ & 9 & 4 & 158 & 13 & $94,6 \%$ \\
\hline $\begin{array}{c}\text { Cessenon } \\
\text { (Héroult) } \\
18-\mathrm{VII}-62 \\
n \circ 27\end{array}$ & 6 & 22 & 101 & 24 & $94,4 \%$ \\
\hline $\begin{array}{c}\text { Riez } \\
\text { (Bosses-Alpes) } \\
14-\text { VII- } 64 \\
\text { no } 151\end{array}$ & 7 & 5 & 54 & 9 & $88,5 \%$ \\
\hline $\begin{array}{c}\text { lo Borie - Nouvelle } \\
\text { (Hérault) } \\
\text { 12-VII-64 } \\
n^{\circ} 201\end{array}$ & 0 & 1 & 258 & 26 & $100 \%$ \\
\hline $\begin{array}{c}\text { Cessenon } \\
\text { (Herault) } \\
26-\mathrm{VII}-64 \\
\text { no } 249 \\
\end{array}$ & 34 & 8 & 17 & 5 & $33,3 \%$ \\
\hline $\begin{array}{l}\text { la Borie-Nouvelle } \\
\text { (Héroult) } \\
28 \text {-VII-65 }\end{array}$ & 0 & 0 & 425 & 12 & $100 \%$ \\
\hline $\begin{array}{l}\text { Roquedur-le-Bas } \\
\text { (Gard) } \\
4-\mathrm{VII}-65\end{array}$ & 0 & 4 & 227 & 18 & $100 \%$ \\
\hline $\begin{array}{c}\text { le-Mas-Supérieur } \\
\text { (Gard) } \\
9 \text {-VII-65 }\end{array}$ & 0 & 0 & 81 & 8 & $100 \%$ \\
\hline
\end{tabular}

TABLEAU VII. - Quelques résultats de captures par la méthode manuelle nocturne à la lampe. Noter la prédominance de Phlebotomus ariasi

\section{II. - Arguments directs}

\section{Infestation spontanée.}

Pour l'instant, la recherche de l'infestation spontanée des Phlébotomes n'a donné aucun résultat. Ainsi, sur 737 Phlebotomus ariasi + inoculés au Hamster, de 1963 à 1965, aucun n'a montré d'infestation leishmanienne.

Cette négativité qui, pensons-nous, n'entame rien à notre hypothèse de travail, relève de deux causes essentielles : 
La première, et vraisemblablement la plus importante, doit être recherchée dans la rareté absolue de l'infestation, rareté sur laquelle insistent la plupart des chercheurs. Ainsi, au Brésil, M. L. Deane n'obtient que trois positivités sur 1.017 Phlebotomus longipalpis étudiés (1954). Au Portugal, J. Fraga de Azevedo dissèque 2.644 Phlebotomus perniciosus pour trouver un seul exemplaire infesté (1954). De même, en Sicile, S. Adler et O. Theodor dépistent une infestation sur 771 Phlebotomus perniciosus.

Une deuxième raison tient aux conditions d'échantillonnage. La majorité des Phlébotomes inoculés à l'occasion de notre enquête, ont été récoltés au hasard des prospections, et non dans les foyers leishmaniens en activité. Or, dans les statistiques de S. Adler et O. Theodor (1931) l'unique exemplaire infesté provenait d'une maison où avait été dépisté un cas de Leishmaniose viscérale. Dans celles de H. E. Shott et coll. (1930), où le nombre d'infestation atteint 3,1 \% (Phlebotomus argentipes), les récoltes avaient eu lieu systématiquement dans les habitations de sujets atteints de Kala-Azar.

\section{Réceptivité expérimentale.}

Les arguments indirects, c'est-à-dire chorologiques, écologiques et éthologiques impliquant nettement Phlebotomus ariasi, il était logique d'étudier concuremment la réceptivité expérimentale de cette espèce.

On sait les problèmes que pose une telle étude. Ils relèvent non seulement des difficultés d'élevage et d'entretien en insectarium, mais, plus encore, du peu d'attraction que manifeste au laboratoire Phlebotomus ariasi à l'égard d'hôtes pourtant très exploités dans les conditions naturelles.

Il était certes possible de provoquer artificiellement l'infestation en faisant ingérer une culture pure de Leptomonas selon la technique de M. Hertig (1927). Mais, outre ses difficultés de réalisation, cette méthode présentait à nos yeux un inconvénient majeur, celui de ne rendre compte que très imparfaitement des conditions naturelles d'infestation.

En effet, en 1963, M. Hertig avait pu montrer à l'aide de souches américaines (Leishmania brasiliensis s.l.) que toutes les espèces de Phlébotomes étaient expérimentalement réceptives. Comme dans ses expériences menées avec des souches de l'Ancien Monde, l'infestation antérieure s'observait dans $80 \%$ des cas.

Les résultats de ces recherches, portant sur un nombre considérable d'individus (800 dans le travail de 1963) revenaient à démontrer indirectement l'importance des facteurs éthologiques dans la transmission des Leishmanioses. En dernière analyse, dans un foyer naturel, le rôle de «vecteur habituel » revenait toujours à l'espèce à la fois la plus abondante et la mieux adaptée aux réservoirs de virus (forte densité, intrication des biotopes, coaptation trophique).

Aussi bien, en 1966, avons-nous abordé le problème par une voie détournée en infestant les Phlébotomes sur un Chien leishmanien, non plus au laboratoire, mais sur le terrain :

« Le 9-7-1966, à La Borie-Nouvelle (Hérault, alt. $500 \mathrm{~m}$, fig. 19), nous installons une moustiquaire-piège sur un terre-plein jouxtant le village. Un Chien leishmanien, présentant 
un pityriasis généralisé avec, par place, lésions de dépilation et de pachydermie (présence de Leishmanies dans la peau de l'abdomen et du museau), est placé dans le piège avec une centaine de Phlebotomus ariasi + capturés sur les murs quelques minutes auparavant. Le lendemain, les Phlébotomes gorgés sont recapturés et mis en pot de conservation. Un dot équivalent, mais placé directement en pots dès la capture, sert de témoin.

Le 21-7, c'est-à-dire 12 jours après, les individus sont disséqués. La tête, détachée du thorax par traction, est écrasée entre lame et lamelle dans une goutte d'eau salée isotonique. La préparation est immédiatement examinée au microscope (object. Leitz 40).

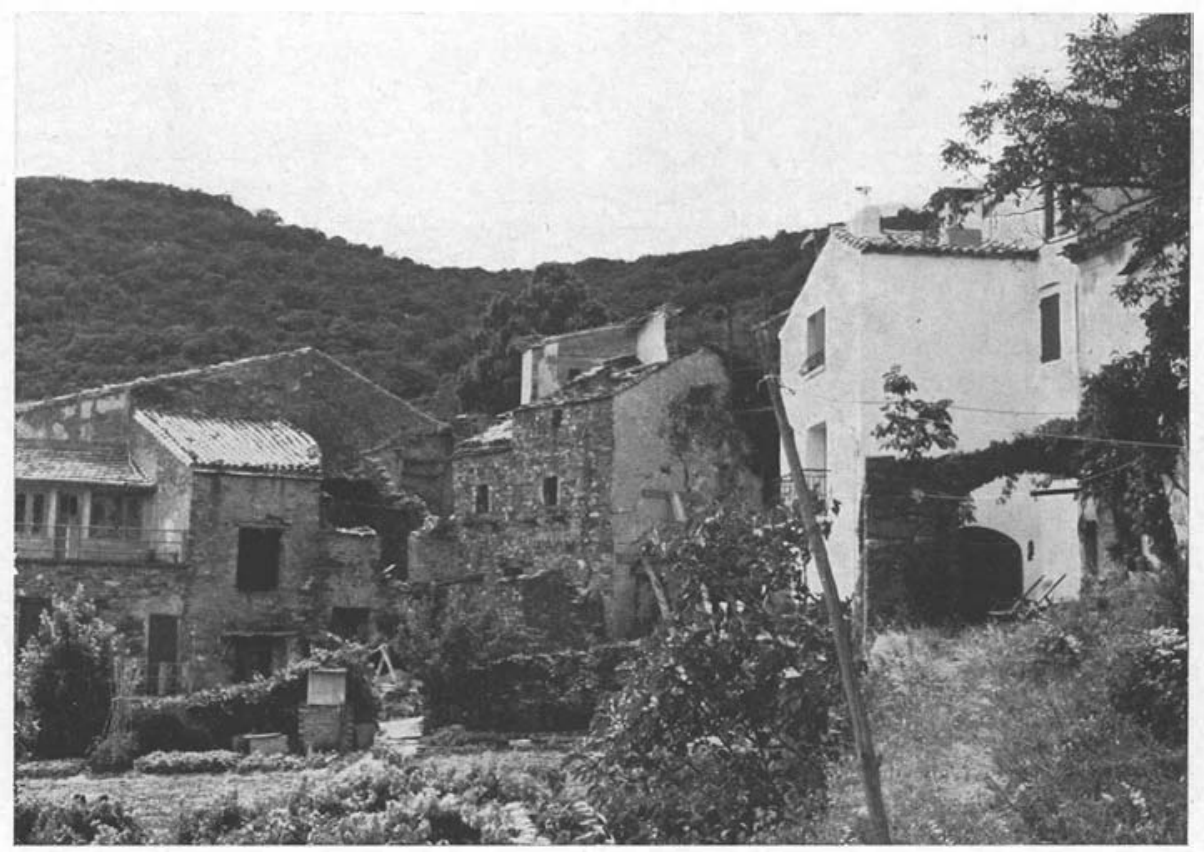

Fıg. 19. - La Borie-Nouvelle (Hérault, $500 \mathrm{~m}$ ). Station optimale de Phlebotomus ariasi. A l'arrière-plan, la Chênaie d'Yeuse montagnarde

Le lot témoin, comportant 61 Phlebotomus ariasi $q$ ne contient aucun parasite. Par contre, dans le lot de 30 Phlebotomus ariasi + gorgés sur le Chien leishmanien, deux individus sont porteurs de Leptomonas typiques, longs et mobiles. »

$$
* *
$$

En définitive, la localisation géographique des Leishmanioses en Languedoc-Roussillon est liée essentiellement à la densité du vecteur principal, Phlebotomus ariasi. Cette densité est elle-même en corrélation étroite avec le milieu (vallées étroites, rocheuses, humides et boisées), et le climat (méditerranéen humide). Elle est maximale en semi-altitude (200 à 500 mètres), sur les versants 
méridionaux du Massif-Central (Cévennes, Montagne-Noire, Espinouse) et des Pyrénées-Orientales (Albères, Roussillon). Dans cette zone, correspondant à l'optimum écologique du vecteur, l'endémie se perpétue grâce aux réservoirs de virus domestiques (Chien) et sauvages (Renard) (19). Au sens épidémiologique, il s'agit d'un véritable « foyer naturel ».

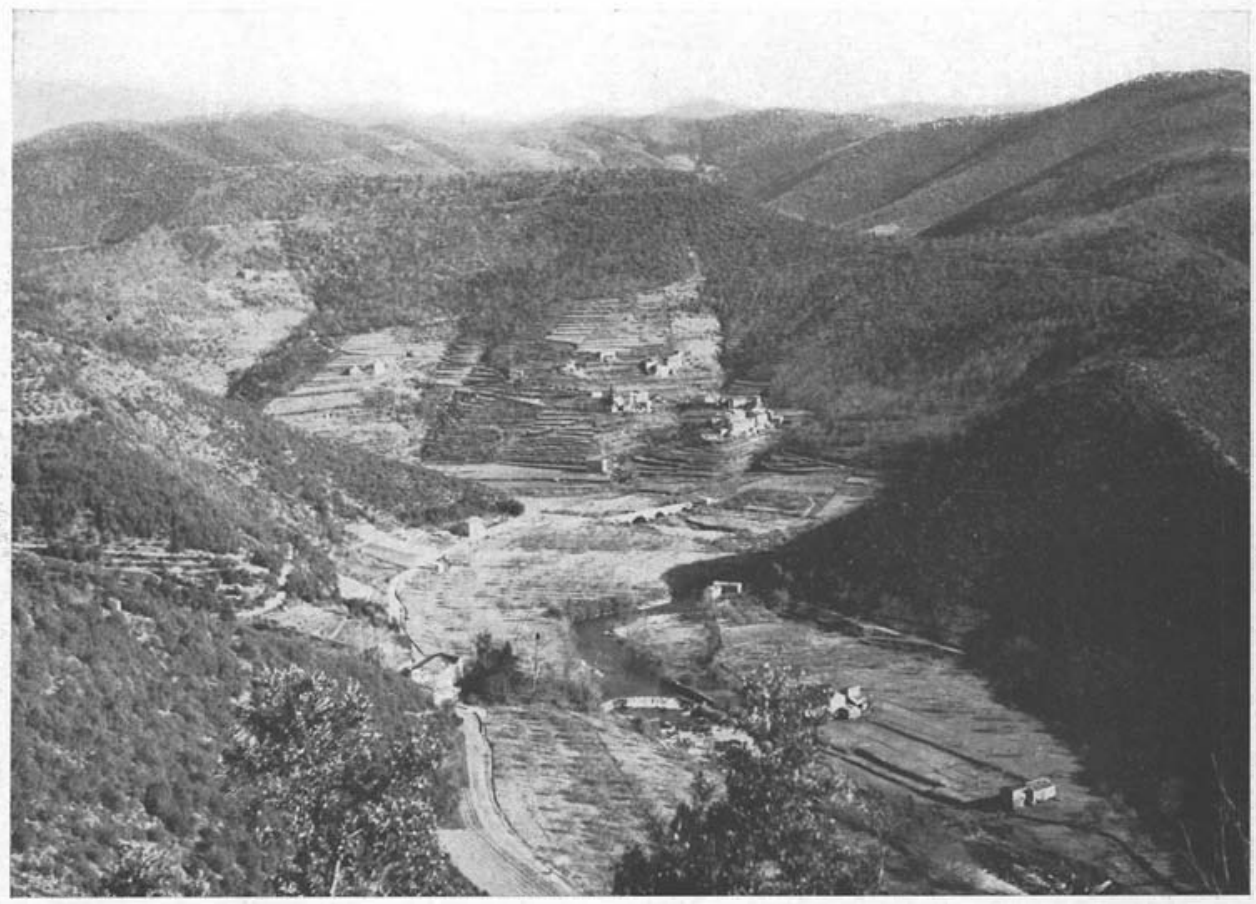

FIg. 20. - La haute vallée de l'Hérault aux environs de Valleraugue (Gard), station optimale de Phlebotomus ariasi. Le long des berges, prairies de fauche plantées de pommiers. Sur les pentes, la forêt mixte de Chênes verts (Quercus ilex) et de Chênes blancs (Quercus pubescens). Par place, la Châtaigneraie.

\section{Résumé}

Dans le présent mémoire, les auteurs exposent les résultats de leur enquête écologique sur les Phlébotomes, vecteurs des leishmanioses dans le sud de la France. Successivement sont étudiées les méthodes d'échantillonnage, la répartition et l'éthologie des cinq espèces présentes dans les limites géographiques du «foyer naturel »: $P$. minutus Rondani, $P$. papatasi (Scopoli), P. mascittii Grassi, $P$. perniciosus Newstead et $P$. ariasi Tonnoir. Par son type de distribution, sa densité et son comportement (anthropozoophile et exo-endophile), $P$. ariasi peut être considéré comme le principal, sinon l'unique vecteur des Leishmanioses humaines dans la région Languedoc-Roussillon.

(19) L'infestation spontanée du Renard (Vulpes vulpes) fera l'objet d'une prochaine note. 


\section{REMERCIEMENTS}

La présente enquête a pu être réalisée grâce à l'aide de l'I.N.S.E.R.M. et de l'O.M.S. Nous remercions particulièrement les responsables des sections «Epidémiologie » qui n'ont cessé de nous manifester leur active sympathie, les Professeurs N. Ansari, P. Chassagne et Martin-Bouyer.

Nos remerciements vont également aux nombreuses personnes qui ont participé à nos recherches ou nous ont aidé de leurs conseils : Mesdames H. Combes, O. de Firmas et S. Seyfor. Mlles F. Deltour et T. Roviralta. MM. E. Abonnenc, J.-P. Baudouy, J.-P. Castells, J. Coudert, L.-P. Knoepffler, J. Mouchet et O. Theodor.

\section{Bibliographie}

Abonnenc (E.) et Rioux (J.-A.), 1961. - Contribution à l'étude des Phlébotomes (DipteraPsychodidae) du Nord-Tchad. In :Mission épidémiologique au Nord-Tchad, Prohuza, Paris : $30-52$.

Adler (S.) et Theodor (O.), 1931. - Investigations on Mediterranean Kala-Azar. III - The sandflies of the Mediterranean Basin. Distribution and bionomics of sandflies in Catania and District. Proc. Roy. Soc. London, 108: 464-480.

BaUdrimont (A.), 1946. - Nouvelles observations sur la présence de Phlebotomus ariasi Tonnoir, 1921 à Saint-Sauveur (Hautes-Pyrénées). Bull. Soc. Lin. Bordeaux, 5 : 204207.

Blanc (G.) et Caminopetros (J.), 1930. - La transmission du Kala-Azar méditerranéen par une Tique, Rhipicephalus sanguineus. C.R. Ac. Sc., 191: 1162-1164.

—, —, 1937. - Quelques expériences sur la transmission du Kala-Azar par la Tique du Chien Rhipicephalus sanguineus. C.R. Soc. Biol., 107: 1493-1495.

Callot (J.), 1950. - Présence de Phlebotomus larroussei en Alsace. Ann. Paras. Hum. Comp., 25: 112 .

Colas-Belcour (J.) et Abonnenc (E.), 1948. - Contribution à l'étude de Phlebotomus (Prophlebotomus) minutus Rondani en France. Bull. Soc. Path. Exot., 41 : 222-225.

— et TisseuIL (J.), 1936. - Nouvelle contribution à la répartition des Phlébotomes en France. Rev. Path. Comp. Hyg. Gén., 36: 117-123.

Corradetti (A.), Sacca (G.) et Neri (I.), 1956. - Studi epidemiologici sul Kala-Azar nel promontorio Garganico (Puglie, Provincia di Foggia). Rendi. Inst. Sup. Sani., 19: 1230-1236.

Coudert (J.), 1947. - Présence de Phlebotomus papatasi (Scopoli, 1786) à Gigondas (Vaucluse). Bull. Soc. Lin., Lyon, 16 : 53-54.

Croset (H.), Rioux (J.-A.), Juminer (B.) et Tour (S.), 1966. - Présence de Phlebotomus ariasi Tonnoir, 1921, en Tunisie et nouvelle mention de Phlebotomus chadlii Rioux, Juminer et Gibily, 1966. Arch. Inst. Pasteur Tunis, 43 : 547-551.

DeANE (L.), 1956. - Leishmaniose visceral no Brasil - Estodos sobre reservatorios e transmissores realizados no estado do Ceara. Serviço Nacional de Educaçao Sanitaria, Rio-de-Janeiro, 162 p. 
Durand-Delacre (R.), 1948. - Quelques observations biologiques sur les Phlébotomes de Béni-Ounif-de-Figuig (Sahara Oranais). Arch. Inst. Pasteur Algérie, 26: 406-430.

— et Memin (Y.), 1953. - Les Phlébotomes des terriers de rongeurs sauvages au Sahara. Arch. Inst. Pasteur Algérie, 31: 320-333.

Fraga de Azevedo (J.), 1948. - Sur la transmission du Kala-Azar méditerranéen. Anais do Inst. Med. Trop., 5 : 269-286.

Gashen (H.), 1945. — Phlébotomes de Suisse. Acta Trop., 2: 137-154.

Gaud (J.), 1947. - Phlébotomes du Maroc. Bull. Soc. Sc. Nat. Maroc, 27 : 207-212.

Hourn (R.), 1963. - Données épidémiologiques et déductions prophylactiques sur les Leishmanioses autochtones en France. Ann. Paras. Hum. Comp., 38 : 379-438.

KIRK (R.) et LewIS (D. J.), 1940. - Studies in Leishmaniasis in the Anglo-Egyptian Sudan. Trans. Roy. Soc. Trop. Med. and Hyg., 33: 623-634.

-, - , 1947. - Studies in Leishmaniasis in the Anglo-Egyptian Sudan. Further observations on the sandflies. Trans. Roy. Soc. Trop. Med. and Hyg., 40: 869-888.

Langeron (M.) et Nitzulescu (V.), 1931. - Phlebotomus larroussei n. sp. nouvelle espèce européenne de Phlébotome. Ann. Paras. Hum. Comp., 9: 72-76.

—, —, 1932. - Révision des Phlébotomes de France. Ann. Paras. Hum. Comp., 10 : 286-294.

Lavier (G.) et Ristorcelli (A.), 1939-1940. — Présence en Seine-et-Marne de Phlebotomus larroussei. Ann. Paras. Hum. Comp., 17 : 375-379.

Legendre (J.), 1929. - La zoophilie des Phlébotomes. Bull. Ac. Med., 102 : 238-240.

Lupasco (G.), Dancesco (P.) et Cheles (N.), 1965. - Contribution à l'étude des espèces de Phlébotomes existant en Roumanie. III. Présence de l'espèce Phlebotomus (Larroussius) major Annandale dans la région de Dobroudgea. Observations sur la biologie et l'écologie de l'espèce. Arch. Roum. Path. Exp. Microb., 24 : 187-194.

Nicoli (R.-M.), 1951. - Phlébotomes de la Corse cristalline. Etude biométrique et critique du Phlebotomus perniciosus Legeri (J. Mansion, 1913) (Nematocera Psychodidae). Bull. Soc. Path. Exot., 44 : 495-509.

-, 1952. - Phlébotomes de la Corse : biologie du Phlebotomus perniciosus Legeri (J. Mansion, 1913). C.R. Soc. Biologie, 146 : 578-580.

— et Nicoli (J.), 1960. — L'apparition des Phlébotomes adultes en Corse au cours de l'été [Larroussius perniciosus Legeri (J. Mansion, 1913)] Données bioclimatologiques. Bull. Soc. Ent. France, $65: 22-26$.

Nitzulescu (V.), 1930. - Sur le Phlebotomus ariasi Tonnoir, 1921, sa présence en France. Ann. Paras. Hum. Comp., 8 : 530-539.

Malamos (B.), 1938. - Versuche mit Leishmanias. Versuche der kala-azar übertragung durch Zecken (Rhipicephalus sanguineus). Arch. für Schiffs und Tropen Hygien., 42: 22-23.

Parrot (L.), 1935. - Nouvelles recherches sur l'évolution de Leishmania tarentolae chez Phlebotomus minutus Rondani. Bull. Soc. Path. Exot., 28 : 958-960.

-, Donatien (A.) et Lestoquard (F.), 1933. - Notes et réflexions sur la biologie de Phlebotomus perniciosus Newstead en Algérie. Arch. Inst. Pasteur Algérie, 11: 183-191.

— et Durand Delacre (R.), 1948. - Notes sur les Phlébotomes. LX. Quelques remarques sur les Phlébotomes des terriers de rongeurs du sud Oranais. Arch. Inst. Pasteur Algérie, $26:$ 402-405. 
Pringault (E.), 1920. - Les Phlébotomes dans la région marseillaise. Bull. Soc. Path. Exot., $13: 809-810$.

Quate (L. W.), 1964. - Phlebotomus sandflies of the paloich area in the Sudan (DipteraPsychodidae). J. Med. Ent., 1: 213-268.

Raynal (J.) et Le GaC (P.), 1933. - Etude sur plusieurs lots de Phlébotomes capturés dans différents quartiers de Marseille. Ann. Paras. Hum. Comp., 11 : 249-267.

Rioux (J.-A.), Golvan (Y.-J.), Deltour (F.) et Houin (R.), 1962. - Nouvelles stations Françaises de Phlebotomus ariasi Tonnoir, 1921, P. perniciosus Newstead, 1911, P. mascittii Grassi, 1908 et P. minutus Rondani, 1843. Ann. Paras. Hum. Comp., 38 : 630-632.

-, Coluzzi (M.), Bain (O.) et Baudouy (J.-P.), 1964. - Présence de Phlebotomus ariasi Tonnoir, 1921, en Italie du Nord. Bull. Soc. Path. Exot., 57 : 966-971.

-, Juminer (B.), Kchouk (M.) et Gibily (H.), 1964. - Récolte de Phlébotomes dans la région de Gafsa. Arch. Inst. Pasteur Tunis, 41: 365-371.

Sicart (M.), Escande (F.), et Ruffié (J.), 1958. - Les Psychodidae du sous-bassin aquitain. Bull. Soc. Hist. Nat. Toulouse, 93 : 70-72.

Simitch (T.), 1932. - Présence à Skoplje d'une nouvelle variété de Phlebotomus perniciosus. Ann. Paras. Hum. Comp., 10: 431-434.

Tonnorr (A.), 1921. - Une nouvelle espèce européenne du genre Phlebotomus (Phlebotomus ariasi). Ann. Soc. Ent. Belgique, 61 : 53-56.

Toumanoff (C.) et Chassignet (R.), 1954. - Contribution à l'étude des Phlébotomes en Corse. Bull. Inst. Nat. Hyg., 9: 664-687.

Withsel (R. H.) et SHoEppNer (R.F.), 1965. - The atractiveness of Carbon Dioxide to female Leptoconops torrens and Leptoconops kerteszi Kieffer. Mosq. News, 25 : 403-410.

Laboratoire d'Ecologie médicale et Pathologie parasitaire. Faculté de Médecine, 34 -Montpellier 